\title{
A Modeling Methodology for Conflict Control in Multi-Agent Systems ${ }^{1}$
}

\author{
Jiexin Lian and Sol M. Shatz \\ Department of Computer Science, University of Illinois at Chicago
}

\begin{abstract}
Multi-agent systems (MASs) have become an important topic in distributed systems research. These distributed multi-agent systems call for special software modeling methods that explicitly support key system properties such as resource constraints and control of conflicts. Although there have been some system modeling techniques to support MASs design and automatic analysis, most state-of-the-art techniques have not distinguished potential conflicts from real conflicts during the design stage. To solve this problem, we define a new concept, called "potential arcs," which is integrated into colored Petri net modeling to support the modeling of MASs. We present a modeling methodology based on the potential arc concept and illustrate the methodology with a case study, including some associated model analysis.
\end{abstract}

Keywords: Multi-Agent-System, Colored Petri Net, Conflict Resolution, Potential Arc.

\section{Introduction}

During the past decade, software agents and multi-agent systems have attracted significant research and development attention. The idea of agents as autonomous units, which can interact with each other to solve problems, has led to the growth of interests in agent-based design paradigm for software engineering [J01]. As a benefit of agent-based design, a multi-agent system (MAS) has emerged as a concurrent system, which hosts interactive agents to perform tasks or solve problems in a decentralized environment [KG97]. Many of the technologies supporting MASs stem from distributed artificial intelligence (DAI) research [GH97]. MASs are expected to solve problems that may be too large for a single agent, to provide enhanced speed and reliability, and to tolerate uncertain data and knowledge [GH97].

A MAS can be characterized by the following properties [JSM98]: (1) Each agent has incomplete capabilities to solve a problem; (2) there is no global system control; (3) data is decentralized; (4) computation is asynchronous. These properties determine that coordination, as the process by which agents reason about and manage the interdependences among their behaviors and try to ensure that all members of the system act consistently [J96], is critical in the MAS design. Due to the ubiquitous existence of conflicts in MAS, conflict control is essential for coordinated agent behavior. In this paper, we consider the class of conflicts that arise from inter-agent resource independence. A conflict can be defined as follows: the consumption of specific resources by one agent prevents other agents from performing relevant actions due to the fact the multiple agent actions require the same resource. Generally speaking, most state-of-the-art research follows one of the following two approaches to handle run-time resource conflicts:

1) Static avoidance approach. In the design stage of a MAS, each agent is assigned a local plan by an agent designer, and the local plan models the behavior of the agent. After all the agents have been assigned local plans, an algorithm is used to integrate local plans to generate a global plan, which models the behavior of the MAS. Through analyzing local plans, the integration algorithm may request some local plans be updated in order to generate

\footnotetext{
${ }^{1}$ This material is based upon work supported by the U.S. Army Research Office.
} 
conflict-free global plans - a global plan being conflict-free means that no run-time conflict is possible to occur in the MAS described and developed based on this global plan. A number of publication results describe research works using this approach [BL99, BL01, MT96, RH03, RHM03]. For example, [RH03, RHM03] followed this approach to design a conflict resolution algorithm for air traffic management. In [RH03, RHM03], an aircraft is modeled as an agent, and the airspace is partitioned into cells and each cell is modeled as a resource unit. It is required that each cell can only be occupied by at most one aircraft at any moment. A local plan for an aircraft is modeled as a timed directed graph, where vertices represent cells (labeled with cell name) and the arc represents the adjacency relation between two cells. Each arc is also labeled with a number to represent the travel time between two cells. Following its local plan, an aircraft is scheduled to travel from the initial cell to the destination cell. The global plan in [RH03, RHM03] is simply a union of local plans. For example, Fig. 1.1 is a global plan including local plans for two aircrafts, $R$ and $S$. Aircraft $R$ will fly from Detroit to Miami via Chicago and Atlanta, and aircraft $S$ will fly from Pittsburg to Los Angels via Chicago and Salt Lake City. Here a cell is represented by a US city. If both aircrafts take off at the same time, they will both need to occupy cell Chicago after 1 time unit, which leads to a conflict because each cell can be occupied by at most one aircraft at anytime. Such a conflict may be detected after the global plan is analyzed by an algorithm, and the conflict resolution algorithm will mandate either $R$ or $S$ to change its local plan to make the global plan conflict-free. The drawback of the static avoidance approach is that the local plans are dependent on each other in order to guarantee that the global plan remains conflict-free. As a result, an individual agent cannot be designed and revised independently.
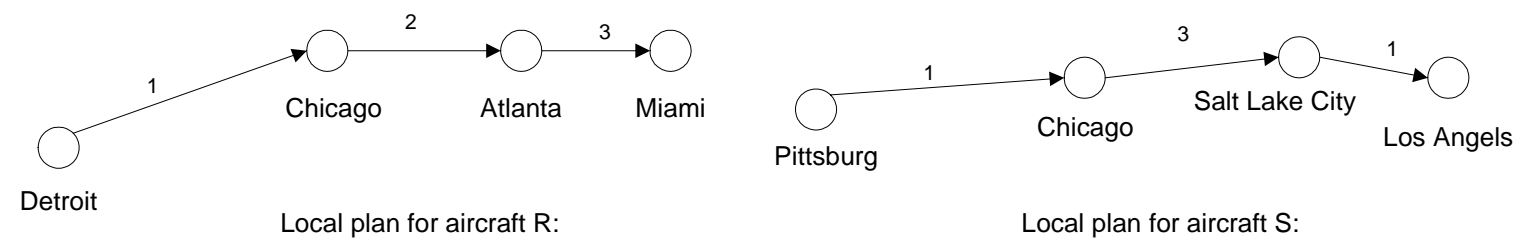

Fig. 1.1 The Global Plan for a MAS Model Including Two Aircraft $R$ and $S$

2) Negotiation approach. Individual agents are designed independently, and conflict control is delayed until runtime by negotiation. When a run-time conflict is detected, ${ }^{2}$ involved agents may need to pause their current activities, and negotiate using a predefined protocol to resolve the conflict. Waiting for negotiation results can be expected to make the agents idle (blocked) during the runtime, and some conflicts might not be resolved at all. Two examples of research work that follow the negotiation approach are [LT05, S94]. Note that the actual air traffic management currently uses the negotiation approach. When facing conflict for airspace cell occupation, two aircrafts negotiate through an air traffic control center (ATC) so that one aircraft will abandon the original plan and be granted a new plan. This new plan is computed by ATC based on currently available resources and flight plans of other aircrafts.

A disadvantage of the negotiation approach is that it suffers from low temporal efficiency due to the temporal cost of negotiation. For example, negotiation with ATC will incur flight delays because pilots have to await the response from ATC. Also, since some plans are generated dynamically after negotiation and are unknown in the design stage, the designer cannot perform comprehensive agent behavior analysis to achieve efficient resource

\footnotetext{
${ }^{2}$ Conflict detection algorithms are outside the focus of this paper.
} 
utilization. The negotiation approach is currently adopted by air traffic management because delays are tolerable and conflicts are relatively rare due to the large capacity of the airspace. However, for those multi-agent systems that favor temporal efficiency (For example, parallel processing system) and/or efficient use of rare resources, the negotiation approach can be undesirable.

Generally speaking, techniques based on the static avoidance approach achieve better temporal efficiency and resource utilization, but are cumbersome in dealing with large-scale systems due to the fact that agents cannot be designed independently. The cause for such design dependence is that such techniques only model the static properties of the MAS, and these static models are not designed to explicitly simulate the execution of a MAS. Thus, algorithms applied to static models must identify conflicts that are possible at run-time, without considering any runtime events that would trigger such conflicts, and the algorithms must eliminate these conflicts by imposing various constraints during the modeling stage. These constraints prevent the local plans from being designed independently. But, a run-time conflict only occurs when it is triggered by a run-time event corresponding to some competition for an external resource, although the actual occurrence of such an event is unknown in the MAS design stage. For example, consider two agents $A 1$ and $A 2$ that model two different airplanes, and each airplane requires a runway for landing. A conflict occurs only when both airplanes request to use the same runway at the same run-time moment. Using a static model, which does not support run-time events, we can avoid the conflict only by creating agent models that assign different runways for different airplanes. In this case, neither $A 1$ nor $A 2$ can be designed independently without the risk of violating the constraint that the design be conflict free. The key point here is that, to overcome the limitation on agent design independence, we need a new modeling approach that distinguishes a potential conflict from a real conflict by considering the appropriate resource-competition event that triggers the conflict. Supporting such a distinction in the design stage can avoid unnecessary potential conflict elimination. In contrast to the static avoidance approach, we call such a modeling approach a dynamic avoidance approach, which distinguishes the representation of potential conflicts from the representation of real conflicts.

To create models that adhere to the dynamic avoidance approach, we define a new feature called a potential arc that is used to extend traditional Colored Petri Net (CPN) models to support agent behavior modeling. The potential arc concept was first discussed in [JS06]. Potential arcs serve to extend the traditional CPN model with an explicit support for distinguishing the representation of potential conflicts and real conflicts, and thereby avoid the need of eliminating all potential conflicts in the design stage. As a result, we can achieve independence in local plan designs. We select CPN as the modeling tool because CPN-based MAS models can support the simulation of dynamic execution of a MAS and the token driven mechanism in CPN can be used to describe the event triggering mechanism of conflicts. Also, the existence of many CPN-based agent modeling techniques [H95, MW97, WH02, HM04, XS03, HB04, WH04] indicates that CPN is an effective tool in MASs modeling.

Based on the potential arc concept, we present a MAS modeling methodology. In order to provide a foundation for the automation of MAS modeling, we extend the formal definition of Colored Petri Net (CPN) [J97] to design a Potential Colored Petri Net (PCPN). The design of our modeling methodology embraces the principle of "separation of concerns" in the agent-oriented design, and focuses on modeling the possible behaviors of the MAS. As such, this work is complementary to other research efforts that model other aspects of a MAS [WH02, WH04, 
XS03, HM04]. The modeling methodology will result in a global plan for the MAS and an individual plan for each agent. Also, an individual plan will include multiple paths for agent actions. When an agent is executed according to a path in its local plan and a conflict arises, an alternative path can be selected from the local plan in order to continue the execution. Because we avoid the need to dynamically compute an alternative path, higher temporal efficiency can be achieved. Also, since the paths of individual agents are determined in the design stage, agent behaviors can be predicted and analyzed to achieve better resource utilization. The selection of alternative paths is decided by the global plan and well controlled by the token-driven mechanism of Petri nets. It is also worth noting that uncertainty and non-determinism are two important features of agent systems. Although uncertainty is not an explicit focus of our technique, our core model can be extended to model uncertainty by using probability functions provided by major Petri net modeling tools, such as CPN Tools. Also, our model only defines the set of possible behaviors for each agent, but does not mandate the execution order of agent actions. This mechanism thus provides the basic support for non-determinism. More complex non-determinism support will be part of future research. State-of-the-art research on explicitly supporting uncertainty and non-determinism can be found in [WR06].

Although the primary focus of this paper is on system modeling, we want to point out that the MAS model can be analyzed at both the local plan level and the global plan level. With regard to local plan analysis, one can ignore potential inscriptions and analyze the local plan as a regular CPN. This is a type of "optimistic" analysis, based on the following assumption: all resources requested by agents are satisfied during the life-cycle of the agent. The local plan analysis results - for example, occurrence graph, boundedness and liveness - still capture the agent's local characteristics; further details on local plan analysis are beyond the scope of this paper. A created global plan is also a CPN. The global plan analysis can be used to capture system-wide properties. Particularly, since potential arcs are used to model the resource demand locally (so that we can distinguish potential conflicts and real conflicts), one can analyze a global plan to study how these real conflicts are resolved and the relevant resource utilization pattern from the global perspective. Section 7.3 illustrates global plan analysis with respect to conflict resolution and resource utilization.

The rest of the paper is organized as follows. Section 2 discusses related research work. Section 3 reviews the preliminary CPN terminology for this paper. Section 4 presents an approach for using Colored Petri Nets for modeling individual agents. Section 5 introduces the concept of potential arcs, and applies potential arcs to extend the modeling capability of Colored Petri Net for the purpose of conflict control. Section 6 presents our overall MAS modeling methodology, which is characterized by a series of algorithms to glue single agent models together for a global view of agent interactions and conflict control. Section 7 presents a case study and Section 8 provides a conclusion and mentions future work.

\section{Related Work}

Applying Petri nets in MAS modeling has been an active research area over the last decade. Many existing research works focus on a high-level interpretation of agents and MASs as Petri net models in a whole [H95, MW97]. For example, in [MW97], Moldt and Wienberg modeled agents as entities like in the object-oriented approach. They used a Petri net representation of the object-oriented concept to redesign Shoham's paradigm of agent-oriented programming [S93]. But, since most of these previous works aim at providing a general Petri net 
based MAS framework, a number of specific modeling issues are not covered. In [MW97], the interface for an agent's communication with external environment is limited to two Petri net places, one for input message, and one for output message. Such a design does not address further details about agent coordination. In contrast, our research addresses specific modeling issues that are of critical importance in agent coordination.

With regard to Petri net based MAS modeling, there also exists research work emphasizing specific modeling aspects [WH02, XS03, XV03, and MN91], but with little emphasis on resource-based agent behavior coordination. Weyns and Holvet use Petri nets for a conceptual modeling of sociality in MASs, and their model is designed in the flavor of supporting integration of agent social skills in a modular way [WH02]. In [XS03], a type of Colored Petri net called G-net is customized to define a so-called "agent-based G-net" that serves as a generic model for agent design. Agent-based G-net focuses on modeling the message processing mechanism of an agent. In [XV03 and MN91], Predicate/Transition (PrT) nets are used to model agent behaviors. For example, [XV03] emphasizes model verification to test whether a multi-agent plan is feasible. Therefore, the models are designed to incorporated critical factors that may impact the model feasibility definition and verification. In contrast to all these work, our approach presents a comprehensive modeling methodology that emphasizes "separation of concerns" in the sense of independently designed local agent plans. In addition, our modeling is aimed specifically at the problem of conflict control and introduces a special type of Petri net arcs to support the conflict modeling.

Two papers that do deal with agent behavior coordination are [HB04] and [WH04]. Hanachi and SibertinBlanc [HB04] introduced a moderator coordination model to manage agent interaction from the organization level. The moderator consists of two parts: a set of Petri net based moderators, each managing a single conversation, and a conversation server, which organizes moderators. Since this work is based on conversations, the interactions of agents are analyzed based on analyzing conversations. In contrast, our work does not draw a clear boundary along conversations, but instead focuses on agent behavior from the view of an individual agent and from the global view of the whole MAS; this provides a modular modeling and analysis framework.

Another area of work close to our research is conflict control in MAS [BL99, BL01, MT96, RH03, RHM03, LT05, S94, WH04, HM04, HB04]. Some contributions [BL99, BL01, MT96, RH03, RHM03] follow the static avoidance approach, while others [LT05, S94, WH04, HM04, HB04] adopt the negotiation approach. As representatives of the first group, [RHM03, RH03] originate from air traffic management, where an aircraft is modeled as an agent while the airspace is partitioned into cells, and each cell is modeled with a resource unit. Each agent in [RHM03, RH03] also has a legal plan, which includes multiple legal paths for realizing the agent goal. However, the plans are modeled as directed graphs and all the potential conflicts involved in legal paths need to be resolved. In contrast, our approach supports the modeling of dynamic resource availability and avoids the need to resolve all the potential conflicts at model-design time. In the work of Barber, et al., PERT/CRM charts [MP95] are extended to model individual plans, and the idea of integrating individual plans is also adopted [BL01]. However, the resulting model is also a static model, requiring the elimination of all potential conflicts. As an example from the negotiation approach, Loutchko and Teuteberg [LT05] design a negotiation protocol based on a series of bilateral negotiations and on a pre-selection procedure that first chooses suitable partners for negotiation. But, like other negotiation-based conflict control solutions, the uncertainty of agent behaviors impedes the effort for efficient 
resource utilization. In contrast, our research shows value in supporting design independence, and creating flexible agent plans with efficient resource utilization.

\section{Colored Petri Net Terminology}

As an extension of basic Petri net [M89], colored Petri net (CPN) models are mathematically precise models, allowing both the structure and the behavior of Petri net models to be described using mathematical concepts. We assume that the reader has some familiarity with basic Petri nets, and we cite some terminologies from [J97] that are important for understanding the details of our modeling notation. Some simple examples are included to illustrate these notations and definitions.

- We use the terms: type, value, operation, expression, variable, binding and evaluation in exactly the same way as these concepts are used in functional programming languages.

- The concept, multi-set [J97] is defined as follows:

A multi-set $m$, over a non-empty set $S$, is a function $m \in[S \rightarrow N]$ ( $\mathrm{N}$ denotes the set of all non-negative integers) which we represent as a formal sum:

$$
\sum_{s \in S} m(s) s
$$

By $S_{M S}$ we denote the set of all multi-sets over $S$. The non-negative integers $\{m(s) \mid s \in S\}$ are the coefficients of the multi-set. $\mathrm{s} \in \mathrm{m}$ iff $\mathrm{m}(\mathrm{s}) \neq 0$.

For example, for set $S=\{a, b\}, m$ is a multi-set, where $m(a)=1$ and $m(b)=2$. This multi-set $m$ is also written as $1^{\prime} a+2 ` b$.

We use $\varnothing$ to denote the empty multi-set. An empty multi-set over $S=\{a, b\}$ is $0^{`} a+0{ }^{`} b=\varnothing$.

- A type is a finite or infinite set, and is used as the meaning in functional programming languages. For example, integer is a type, which is an infinite set, as defined in major functional programming languages.

Example: $P=\{$ weak, strong $\}$ is also a type, which is a finite set with two elements. In CPN, a type is also called a color set. Therefore, when $P$ is used in CPN, it is also called a color set.

- The elements of a type $T$. The set of all elements in $T$ is denoted by the type name $T$ itself. For example, all elements in integer are denoted by the type name integer.

- The type of a variable, $\mathrm{v}$ - denoted by Type(v). The notation Type(v) is also extended to $\operatorname{Type}(A)=\{\operatorname{Type}(v) \mid v \in A\}$, where $\mathrm{A}$ is a set.

Example: Define two variables $x, y$ for color set $P$ given above. Define variable $z$ for color set integer. So we get Type $(x)=P$, and Type $(z)=$ integer. Set $A=\{x, y\}$, then Type $(A)=\{P\}$. Set $A^{\prime}=\{x, y, z\}$, so Type $\left(A^{\prime}\right)=\{P$, integer\}.

- The type of an expression, expr - denoted by Type(expr).

Example: Define expr $=1 x+2 y$ as an expression, Type (expr) $=P$.

- The set of variables in an expression, expr - denoted by Var(expr).

Example: $\operatorname{Var}(\operatorname{expr})=\{x, y\}$. 
- A binding of a set of variables, $V$ - associating with each variable $v \in \operatorname{Var}(\operatorname{expr})$ an element $b(v) \in$ Type(v). Also, by $B(t)$, we denote the set of all bindings for $t$.

Example: Define a binding $b$ for $\{\mathrm{x}, \mathrm{y}\}$ as: $(x, y)=$ (weak, strong).

- The value obtained by evaluating an expression, expr, in a binding, $\mathrm{b}$ - denoted by expr $<b>$.

$\operatorname{Var}($ expr) is required to be a subset of the variables of $b$. And the evaluation is performed by substituting for each variable $v \in \operatorname{Var}(\operatorname{expr})$.

Example: $\exp r<b>=1$ weak +2 strong. Here $\operatorname{Var}(\operatorname{expr})=\{x, y\}$, and the set of variables in $b$ is also $\{x, y\}$, so the evaluation can be performed. If the set of variables in $b$ is $\{x\}$, we can not perform evaluation on expr $<b>$, since $\{x, y\}$ is not a subset of $\{x\}$.

- $B$ denotes the boolean type (containing the elements \{false, true $\}$ ).

- An expression without variables is said to be a closed expression. It can be evaluated in all bindings, and all evaluations give the same value - which we often denote a closed expression by the expression itself. This means that we simply write "expr" instead of the more pedantic "expr $<\mathrm{b}>$."

Example: 1 weak +2 strong is a closed expression.

\section{Single Agent Modeling Based on Colored Petri Nets}

Before discussing conflict control in general, we describe how a CPN model can be used in single agent modeling. For conciseness, an incoming arc refers to the arc from a Petri net place to a transition, while an out-going arc refers to an arc from a transition to a place.

Based on the widely accepted BDI agent model [KG96], an agent has goal, plan, knowledge base, and environment modules. How agents accomplish a goal task in a multi-agent system is usually specified by agent plans built from basic actions of which the agents are capable. Coupled with agent goal, its knowledge-base, environment and reasoning mechanism, agents can autonomously select proper actions to achieve the global goal. Also, performing an action will update an agent's mental state. An agent action may rely on the shared resource to be carried out, and an agent's behavior is characterized by a set of possible actions. With regard to modeling an agent's behavior, we want to emphasize the following characteristics of an agent: An agent is an autonomous entity with several properties and actions. An agent's mental state is a possible assignment of the agent's properties, and we abstract an agent's behavior into a set of transitions between different mental states. An agent action implements some tasks and updates the agent's mental state.

For example, a very simple agent PERSON describes a person's behavior. The person has two properties, isHome and isOffice - to indicate if the person is currently at home or at his or her office. The person also has one action: go-to-work. The action go-to-work moves the person from the home to the office. Both isHome and isOffice have two possible assignments: "YES" or "NO." At a specific run-time moment, isHome equals "YES" means the person is at home, while isHome equals " $N O$ " means the person is not home; similarly for the property isOffice. The tuple (isHome, is Office) represents the agent's mental state. The state (isHome, isOffice) = ("YES", "NO") means the person is home, and the action go-to-work updates the agent's mental state to ("NO", "YES").

We call the model describing an agent's behavior the agent's local plan, and the agent is called the owner of its local plan. The local plan of our agent corresponds to the plan module in the BDI agent model. In the BDI 
model, an agent's properties in the plan module also contain belief state set and goal state set. Goal, knowledge base, environment, belief state set and goal state set are used to describe other aspect for an agent in the BDI model. These components are omitted from our agent model since the technique proposed here focus on agent behavior modeling.

Local plan can be written by various notations, varying from descriptive, semi-formal, to formal notions. In this section, we use CPN to describe a local plan, and our CPN model focuses on describing different actions an agent can take, without concern with the interaction with the MAS environment. We call such a CPN model an action model. Since an action model doesn't consider the interaction with the external MAS environment, we say action model is an isolated version of local plan. In contrast, modeling of an agent in MAS environment needs to include interface in the local plan to support interaction with external environment. Therefore, local plan will be extended from isolated version (action model) to an interactive version, as we will see in Section 5. In the rest of this section, the term local plan refers to both isolated and interactive version of the local plan.

We present some local plan development guidelines here. Applying the following guidelines can create an action model for an agent. For an interactive version of the local plan, we need more guidelines. The details will be discussed in Section 5. In a local plan, one Petri net place is used to model one property of the owner agent, and one transition is used to represent one agent action. For each agent property, the possible assignments to that property contribute to the color set for the corresponding CPN place. Also, a default assignment means there is no colored token in the place. A Petri net arc is used to connect places and transitions, and each arc can carry an inscription. The inscription carried by an incoming (place-to-transition) arc specifies the colored tokens removed from the arc's source place when the arc's destination transition fires. The inscription carried by an out-going (transition-to-place) arc specifies the colored tokens deposited into the arc's destination place when the arc's source transition fires. The local plan can describe its owner agent's run-time behavior. The concept of "running an agent" means the agent performs actions, and the actions update the agent's mental state. From the perspective of our model, an action corresponds to firing a transition in the agent's local plan. Similarly, updating the agent's mental state corresponds to updating the state associated with that local plan.

Take agent PERSON as an example. We define place Home and Office for the agent's two properties isHome and isOffice, respectively; and transition Move for the agent's action go-to-work. The color set for the place Home can have only one element, since isHome has only two possible assignments, and one assignment corresponds to the default assignment of place Home containing no token. We define the color set as \{person\}. If the place Home holds the token "person", then isHome equals "YES." The default assignment for isHome can be defined as "NO." Place Office has the same color set as place Home. We also define arcs (Home, Move) and (Move, Office) in the local plan, and the inscriptions in arcs (Home, Move) and (Move, Office) are both "I person."

In the run-time, taking the action "go-to-work" can be represented by firing the transition Move in the agent PERSON's local plan, which results in the token "person" being removed from place Home and a token "person" being deposited into place Office. According to the mapping rules above, we can design an action model for agent PERSON, as shown in Fig. 4.1 (Initial tokens omitted for now).

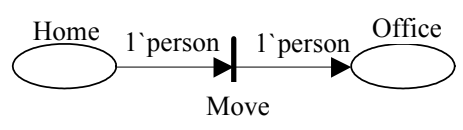


Fig. 4.1. An Action Model for PERSON

Definition 4.1 formally defines an action model in the context of agent modeling. An action model is actually a CPN using the same definition as defined in [J97]. The key point is that we map each element of the CPN definition to a counterpart in the agent modeling domain. Note that Definition 4.1 uses various terms (Type, Var, MS) defined in Section 3.

Definition 4.1: An action model for an agent is a CPN ( $\Sigma, \mathrm{P}, \mathrm{T}, \mathrm{A}, \mathrm{N}, \mathrm{C}, \mathrm{G}, \mathrm{E}, \mathrm{I})$, where:

(1) $\Sigma$ is a finite set of color sets, where each color set is the set of possible assignments for one or more agent properties.

(2) $P$ is a finite set of places, each of which represents an agent property.

(3) $T$ is a finite set of transitions, each of which represents an agent action.

(4) $A$ is a finite set of arcs such that:

$\mathrm{P} \cap \mathrm{T}=\mathrm{P} \cap \mathrm{A}=\mathrm{T} \cap \mathrm{A}=\varnothing$.

(5) $N$ is a node function. It is defined from $A$ into $P \times T U T \times P$. (i.e., $A \rightarrow P \times T U T \times P$ ). Here the node function defines how places and transitions are connected.

(6) $C$ is a color function. It is defined from $P$ into $\Sigma$ (i.e. $P \rightarrow \Sigma$ ). C assigns color set for each place, thereby specifies the possible assignments for each agent property.

(7) $G$ is a guard function. It is defined from $T$ into expressions (i.e. associate an expression with each transition in $T$ ) such that: $\forall \mathrm{t} \in \mathrm{T}:[\operatorname{Type}(\mathrm{G}(\mathrm{t}))=\mathrm{B} \wedge \operatorname{Type}(\operatorname{Var}(\mathrm{G}(\mathrm{t}))) \subseteq \Sigma]$

The guard function casts extra enabling conditions for transitions, and it is not concerned with the key idea we propose in this paper. We use $G_{T}$ to represent the guard function that evaluates to be true for any transition. i.e., $\forall \mathrm{t} \in \mathrm{T}: G_{T}(\mathrm{t})=$ true. For any $\mathrm{CPN}(\Sigma, \mathrm{P}, \mathrm{T}, \mathrm{A}, \mathrm{N}, \mathrm{C}, \mathrm{G}, \mathrm{E}, \mathrm{I})$ in our paper, we assume $\mathrm{G}=G_{T}$.

(8) $E$ is an arc expression function. It is defined from $A$ into expressions (i.e., associate an expression with each arc in $A$ ) such that: $\forall \mathrm{a} \in \mathrm{A}:\left[\operatorname{Type}(\mathrm{E}(\mathrm{a}))=\mathrm{C}(p)_{M S} \wedge \operatorname{Type}(\operatorname{Var}(\mathrm{E}(\mathrm{a}))) \subseteq \Sigma\right]$, where $p$ is the place of $N(a)$.

$E$ specifies the inscription for each arc, and these inscriptions are used to update the agent's mental state after a transition is fired

(9) $I$ is an initialization function. It is defined from $\mathrm{P}$ into closed expressions (i.e., associate an closed expression with each place in $P$ ) such that $\forall \mathrm{p} \in \mathrm{P}:\left[\operatorname{Type}(\mathrm{I}(\mathrm{p}))=\mathrm{C}(\mathrm{p})_{\mathrm{MS}}\right]$.

$I$ defines the initial mental state for the agent.

Based on the formal CPN definition, Petri net can be expressed by two ways, textual notation and graphic diagram. For example, we develop the action model $\mathrm{AM}_{1}$ for the agent PERSON in Fig. 4.1. AM 1 can be expressed by the textual notation given in Fig. 4.2, while Fig. 4.1 is the corresponding graphic diagram. Note that the color set definition is not part of an action model itself but it is a preliminary step for developing the action model (See Figure 4.2). 
$\mathrm{AM}_{1}=\left(\Sigma_{1}, \mathrm{P}_{1}, \mathrm{~T}_{1}, \mathrm{~A}_{1}, \mathrm{~N}_{1}, \mathrm{C}_{1}, \mathrm{G}_{1}, \mathrm{E}_{1}, \mathrm{I}_{1}\right)$, where:

$\Sigma_{1}=\{$ per $\}$. (Note: We have only one color set per in this example)

$P_{1}=\{$ Home, Office $\}$

$T_{1}=\{$ Move $\}$.

$A_{1}=\{r 1, r 2\}$.

$N_{1}=\left\{N_{1}(r 1)=(\right.$ Home, Move $), N_{1}(r 2)=($ Move, Office $\left.)\right\}$.

$C_{1}=\left(C_{1}(\right.$ Home $)=$ per, $C_{1}($ Office $)=$ per $)$.

$\mathrm{G}_{1}=G_{T}$

$E_{1}=\left\{E_{1}(r 1)=1\right.$ person, $E_{1}(r 2)=1$ iperson $\}$.

$I_{1}=\left\{I_{1}(\right.$ Home $)=1$ 'person, $I_{1}($ Office $\left.)=0\right\}$.

Color set definition:

per $=\{$ person $\}$

Fig. 4.2 The textual notation of action model $\mathrm{AM}_{1}$ for agent PERSON

\section{Supporting Conflict Control Using Potential Arcs}

Colored Petri nets, as an abstract model, can be applied to the modeling of a wide range of applications. However, because of this generality, CPN modeling is limited in its ability to provide explicit support for applications in a specific domain. In this section, we discuss how potential arc can extend CPN to provide explicit support for modeling conflicts between agents, with emphasis on the distinction between potential conflicts and real conflicts.

\subsection{Path modeling via potential arcs}

Conflicts are common phenomena in MAS because the enablement of an agent action may involve external resources from other agents. Therefore, we adopt the concept of path to describe the resources relevant to agent actions. Considering the MAS environment, we can extend our description of an agent to include the concept of a path: Within an agent, an action is taken through one of several predefined paths. Associated with each path are an agent action and a set of resources that may be acquired or released. In the runtime, an agent action can be taken along any available path, whose availability is determined by the availability of associated resources (especially external resource). The agent is provided the autonomy to select any available path.

A path is described as a triple ( $a$, iRes, oRes). Here $a$ is the agent action associated with a path, iRes is the resource required to take the action and oRes is the resource to be released after the action is taken. Either iRes or oRes can be empty, or both. For example, (go-to-work, by car, empty) is a path associated with the action go-to-work of the agent PERSON introduced in Section 4. Intuitively, this path can be interpreted as: driving a car is a path to take the action go-to-work. A path is used to explicitly specify the external resource involved to take an action. Multiple alternative paths can be created for the same action.

Now we introduce the concept of potential arc (illustrated by the dashed arcs in Fig. 5.1 and Fig. 5.2) to support path modeling. In the basic CPN model, each arc carries one inscription - we refer to these as regular arcs and regular inscriptions. We now add the capability for an additional inscription to describe explicit need for access to an external resource that is modeled "out-side" of a local plan. This new inscription is called a potential inscription (PI), since the access to the resource is a potential access in terms of the view of the design model. We 
call an arc that includes a potential inscription a potential arc and we call a CPN with potential arcs a Potential Colored Petri Net (PCPN). A potential inscription carried by an incoming (Place to Transition) potential arc specifies the resource unit required to enable the arc's destination transition, while a potential inscription carried by an out-going (Transition to Place) potential arc specifies the resource unit released after the arc's source transition is fired.

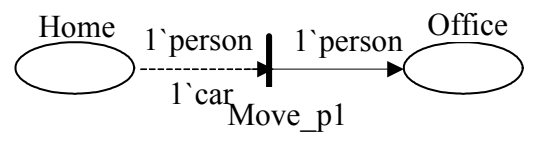

Fig. 5.1. A Potential Arc from Place to Transition

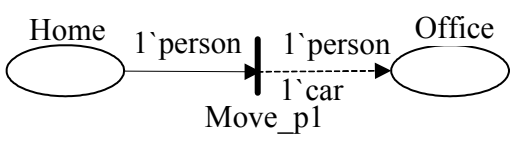

Fig. 5.2. A Potential Arc from Transition to Place

By adopting the concept of potential arc, a local plan can encapsulate the description of paths. Paths are modeled through potential inscriptions. A path ( $a$, iRes, oRes) can be modeled by a triple ( $t$, iPI, oPI). Here $t$ is a transition in an action model associated with action $a$, so we call $t$ an action transition. Both iPI, oPI are PIs. As previously defined, PI is a potential inscription used to describe the external resource involved. Here, iPI is an incoming potential inscription to model iRes, and oPI is the out-going potential inscription to model oRes. Either iPI or oPI can be empty, or both. For instance, the path (go-to-work, by car, empty) can be modeled by the triple (Move, l'car, 0). Moreover, variables are supported in PIs to delay the actual binding of the resources with a path. For example, suppose $a, b$ and $c$ are potential inscriptions and (T, $x, b)$ models a path for an action, where $x$ is a variable that can evaluate to either $a$ or $c$. Thus, the path might be (T, $a, b)$ or (T, $c, b)$; it is purposefully unresolved during the local plan design. By supporting variables, we delay the binding of specific resources in path modeling, and thereby provide design flexibility in MAS modeling.

After the action model has been developed and all the paths are modeled, a PCPN model can be algorithmically generated (See Appendix A1 for the algorithm details, and see Appendix A2 for further comments and examples). The PCPN model integrates agent actions and assorted paths that invoke these actions, so that this model presents a comprehensive description for agent behaviors in a MAS environment. The PCPN model includes one transition for each path associated with an action transition; we call such a transition in the PCPN model a path transition. Consequently, in the PCPN model, a path can be recognized by a triple (path transition, iPI, oPI), and multiple path transitions can exist as the counterparts for the same action transition, if multiple paths are associated with the same action. We call such a PCPN based agent model a path model since it encapsulates paths in the model. Also, we say a path model is the interactive version of a local plan, since it supports the interaction with the external MAS environment.

Fig. 5.1 is a path model extended from Fig. 4.1. Fig. 5.1 contains one path (Move p1, l'car, 0). Here Move $p 1$ is a path transition and the action transition associated with the path is Move in Fig.4.1. Intuitively, the agent modeled by Fig. 5.1 has the potential to fire transition Move. But in order to enable the transition Move, we not only need a token "person" in place Home, but also some other agent from the outside must provide one resource unit "car." Fig. 5.2 is another path model extended from Fig. 4.1. Fig 5.2 contains one path (Move p1, 0, l'car). In Fig. 5.2, we need a token "person" in Home to enable transition Move, and firing the transition would release a 
resource unit (car) that is accessible outside the scope of this local plan. The two path models, Fig. 5.1 and Fig. 5.2, define different ways in which the agent of concern, PERSON, is able to interact with other agents.

Since a path is associated with external resources by potential inscriptions, it carries potential conflicts, because it is possible that some run-time event will make the external resource associated with a path unavailable, and hereby trigger the conflict. Moreover, when an action is associated with a path, it is consequently associated with a potential conflict. At the agent design stage, it is unknown whether a potential conflict will actually evolve into a real conflict, since a real conflict is triggered by a runtime event. Therefore, it is useful to include several paths for each agent action in an agent's local plan. In the runtime, real conflicts may cause some paths to be blocked, but, as long as an unblocked path exists, the corresponding agent action can be taken, resulting in a conflict-free behavior in the MAS environment. In this way, we tolerate potential conflicts in the agent design stage. Only if all the paths are blocked at the same time, the associated action cannot be taken. However, it is reasonable to believe that the possibility of blocking all the paths decreases significantly when more paths are associated with the same action.

Definition 5.1 formally defines PCPN as a 9-tuple $P C P N=(\Sigma, P, T, A, N, C, G, S, I)$. Compared with Jensen's original CPN definition [J97] $C P N=(\Sigma, P, T, A, N, C, G, E, I)$, the arc expression function $E$ in $C P N$ is now replaced by $S$, where $S$ is a pair, $S=\left(E_{r}, E_{p}\right)$. Both $\mathrm{E}_{\mathrm{r}}$ and $\mathrm{E}_{\mathrm{p}}$ are arc expression functions as $E$ in $\mathrm{CPN}$. $\mathrm{E}_{\mathrm{r}}$ is defined as the regular arc expression function, while $\mathrm{E}_{\mathrm{p}}$ is defined as the potential arc expression function. Also, $\Sigma$ now turns to a pair, $\Sigma=\left(\Sigma_{\mathrm{r}}, \Sigma_{\mathrm{p}}\right)$. $\Sigma_{\mathrm{r}}$ and $\Sigma_{\mathrm{p}}$ are both finite sets of color sets. The role of $\Sigma$ is to provide vocabulary for arc inscription specification. PCPN needs two finite sets of color sets in its vocabulary $\Sigma$, because it is designed to deal with both regular inscription domain and potential inscription domain, where vocabulary in these two domains is not necessary to be dependent on each other. Each place in $P$ is assigned a color set from $\Sigma_{\mathrm{r}}$. Although a regular arc expression needs to be evaluated as a multi-set over the color set of its associated place, the associated place imposes no restriction on the potential arc inscription.

Definition 5.1: A PCP-net is a 9-tuple PCPN $=(\Sigma, \mathrm{P}, \mathrm{T}, \mathrm{A}, \mathrm{N}, \mathrm{C}, \mathrm{G}, \mathrm{S}, \mathrm{I})$ where:

(1) $\Sigma=\left(\Sigma_{r}, \Sigma_{p}\right)$, where $\Sigma_{r}$ is a finite set of color sets, and $\Sigma_{p}$ is a finite set of color sets

(2) $P$ is a finite set of places.

(3) $T$ is a finite set of transitions.

(4) $A$ is a finite set of arcs such that:

$P \cap T=P \cap A=T \cap A=\varnothing$.

(5) $N$ is a node function. It is defined from $A$ into $P \times T \cup T \times P$. i.e. $(A \rightarrow P \times T \cup T \times P)$

(6) $C$ is a color function. It is defined from $P$ into $\Sigma_{r}$ (i.e. $P \rightarrow \Sigma_{r}$ ).

(7) $G$ is a guard function. It is defined from $T$ into expressions (i.e. associate an expression with each transition in $T$ ) such that $\forall \mathrm{t} \in \mathrm{T}:\left[\operatorname{Type}(\mathrm{G}(\mathrm{t}))=\mathrm{B} \wedge \operatorname{Type}(\operatorname{Var}(\mathrm{G}(\mathrm{t}))) \subseteq\left(\Sigma_{r} \cup \Sigma_{p}\right)\right]$.

Similar with action model, the guard function here is not concerned with the key idea we propose in this paper. Therefore, we assume $G=G_{T}$ for all the PCPN in this paper. 
(8) $S$ is a pair $\left(E_{r}, E_{p}\right)$, where $E_{r}$ and $E_{p}$ are both arc expression functions - as in the CPN definition (the arc expression function in Def 3.1) - such that $\forall \mathrm{a} \in \mathrm{A}:\left[\operatorname{Type}\left(\mathrm{E}_{\mathrm{r}}(\mathrm{a})\right)=\mathrm{C}(p)_{M S} \wedge \operatorname{Type}(\operatorname{Var}(\mathrm{E}(\mathrm{a}))) \subseteq \Sigma_{r}\right]$ and $\forall \mathrm{a} \in \mathrm{A}:\left[\operatorname{Type}\left(\operatorname{Var}\left(\mathrm{E}_{\mathrm{p}}(\mathrm{a})\right)\right) \subseteq \Sigma_{p}\right]$,

where $\mathrm{p}$ is the place of $\mathrm{N}(\mathrm{a})$.

(9) I is an initialization function. It is defined from $P$ into closed expressions (i.e., associate an closed expression with each place in $P$ ) such that $\forall \mathrm{p} \in \mathrm{P}:\left[\operatorname{Type}(\mathrm{I}(\mathrm{p}))=\mathrm{C}(\mathrm{p})_{\mathrm{MS}}\right]$.

As we can see from the definition, every arc is associated with two inscriptions. For an regular $\operatorname{arc} a$, we define $E_{p}(a)=0$. In this sense, regular arc is a subset of potential arc, and CPN is thereby a subset of PCPN, since CPN only contains regular arcs.

Like a CPN model, a PCPN model can be expressed as both textual notation and graphical diagram. We show the path model PM (it is a PCPN model) of PERSON in Fig. 5.3 and Fig. 5.1. Fig. 5.3 is the textual notation for PM, while Fig. 5.1 is the graphical diagram. As we can see from Fig. 5.3, the path model will inherit the places $\left(P_{A M}\right)$ and initialization function $\left(I_{A M}\right)$ from the action model. Also, $\Sigma_{p}=\{$ Vehicle $\}$ is added and potential inscription in $E_{p r}$ will be specified over $\Sigma_{p \text {. }}$

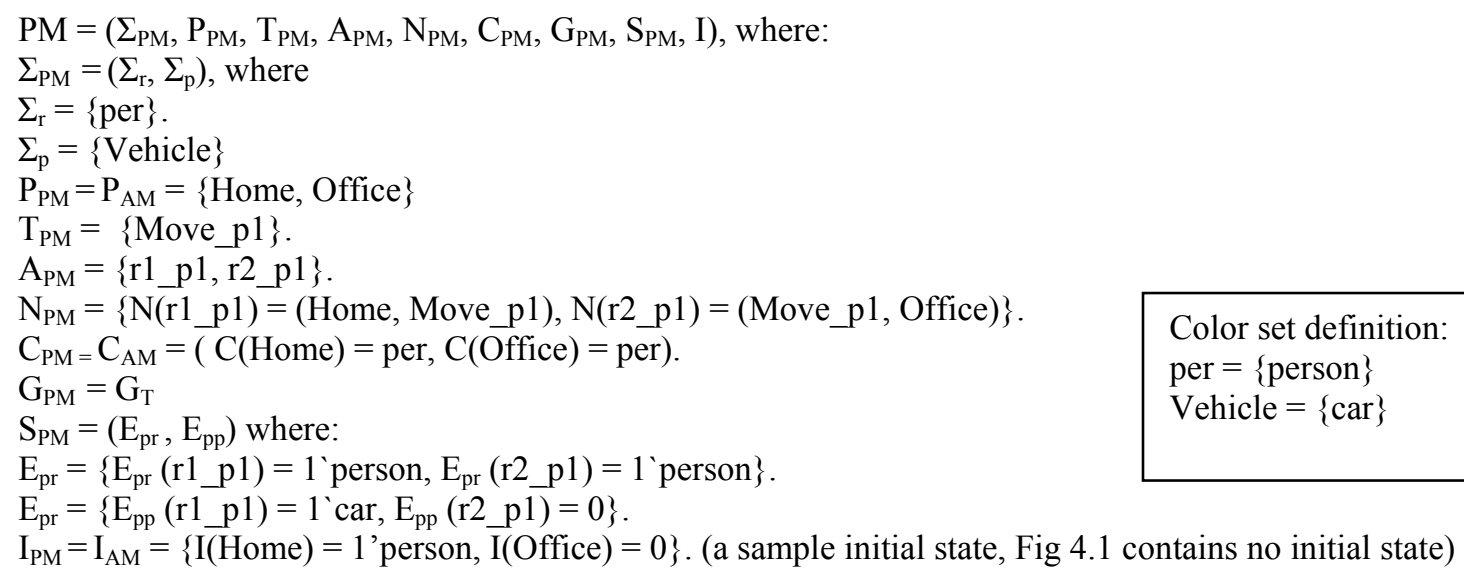

Fig. 5.3. The Textual Notation for the Path Model PM of Agent PERSON

\subsection{Interpretation of potential arcs to support global plan modeling}

With regard to the design stage of an agent's local plan, the designer can use potential arcs to describe paths for agent actions, without concern for whether resources will be available at run-time. After creating the individual path model for each agent of the MAS, the path models will be concatenated to form a global plan. This concatenation process depends upon an appropriate interpretation for the potential arcs that appear in the path models. A potential arc is interpreted as follows: For each potential arc, we create a place node (called a resource place) outside the local plan to produce or consume the resource unit specified by the potential inscription. Fig. 5.4 and Fig. 5.5 show examples of how a potential arc in a local plan is interpreted in the global plan. As we can see, Fig. 5.4 shows how the potential arc in Fig. 5.1 is interpreted in a global plan. The potential arc (Home, Move_p1) in Fig. 
5.1 is replaced by a regular arc, and place $R 1$, outside the local plan model, is added to control the enablement of Move $\_$1. R1 needs to produce the token "car" so that transition Move $p 1$ can be enabled. Similarly, Fig. 5.5 shows how the potential arc in Fig. 5.2 is interpreted in a global plan.

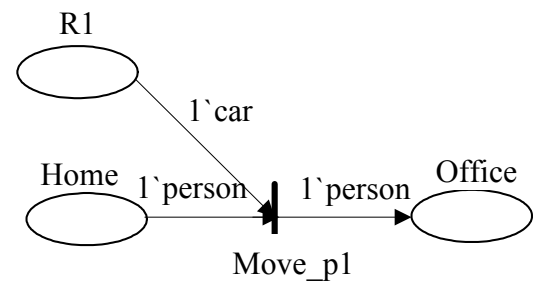

Fig. 5.4 The Interpretation of Fig. 5.1

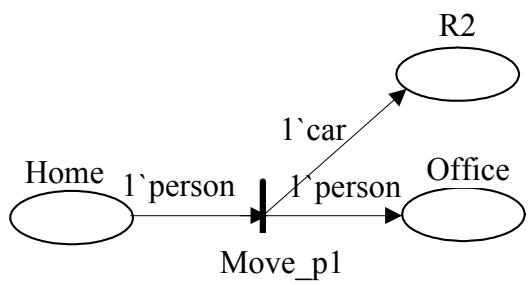

Fig. 5.5 The Interpretation of Fig. 5.2

The concatenation procedure interprets all the potential arcs from different path models by the semantics above, and also synthesizes a unit, modeled as a CPN, called a coordinator. The final step in the global plan design involves connecting the coordinator with all the path models to form the global plan. Through concatenation, the local plans are integrated into a global plan. The global plan inherits all the resource places from the coordinator and all the places and transitions from each local plan, and describes the behavior of the whole MAS. Therefore, the global plan incorporates the resource utilization descriptions from individual agents to allow for the representation of potential conflicts and real conflicts.

According to the definition given in [LR78], in a CPN, two Petri net transitions are said to be in a structural conflict if they have a common input place. Two transitions in a structural conflict are said to be in a behavioral conflict if there is a reachable marking in which both transition are enabled but firing one disables the other. Based on these definitions of structural conflict and behavior conflict, we can define potential conflict and real conflict as follows:

Potential conflict: In a MAS, there exists a potential conflict iff there exists a structural conflict in the global plan, where the two transitions in the structural conflicts are inherited from two different local plans and the common input place is a resource place.

Real conflict: In a MAS, a potential conflict is said to be in a real conflict if there is a reachable marking in which both transitions in the potential conflict are enabled but firing one disables the other.

An important observation is that with our approach, an agent's local plan can be modified in a way that is transparent to other agents. This is because a modified path model can be reconnected to an existing global plan as long as the potential arcs of the modified path model remain the same as in the pre-modified path model. Detail on the concatenation procedure will be explained in Section 6.

\section{A Modeling Methodology}

Based on the discussion about PCPN based MAS modeling in Section 5, we present a potential arc based modeling methodology to develop a MAS system. Generally speaking, the modeling methodology creates local plans first and then concatenates local plans to form a global plan. The global plan, which is a regular CPN, can be analyzed by CPN Tools [CPN 06] for simulation and model checking.

From the software life-cycle point of view, the development of a MAS should cover the following phases: requirement capture, design, implementation and deployment. During the requirement capture phase, the scenario to 
model is analyzed so that the stakeholders can be identified. Furthermore, the behavior of each stakeholder is defined and each stakeholder is assigned an agent role in the MAS, based on its behavior pattern. During the design stage, agent models will be created for each role in the MAS. The implementation phase turns the agent models into executable codes. After testing, executable codes will be deployed. State-of-the art research has provided several methodologies to cover one or more phases in MAS development, such as Gaia[WJ00], MaSE[DW01] and Tropos [BG04].

Our methodology focuses on the design phase, since providing conflict control support at this stage can relieve the effort of conflict resolution in later phases. Integrating our design phase methodology with other approaches that deal with other phases in MAS development life-cycle can develop a comprehensive methodology which covers the whole MAS development life-cycle. [FG05] provides a comprehensive MAS development methodology by integrating different existing methodologies.

For the design phase, a preliminary step in the successful design of agent models is to define a universal vocabulary that can be understood in the whole MAS system. Clarifying the vocabulary can avoid many problems typically caused by the assumption that different agents in the MAS can understand each other automatically [CY03]. The role of vocabulary in MAS is similar to the role of human language in human society. In human society, two people speaking two different languages cannot communicate with each other. Likewise, in the agent society, two agents using different vocabularies cannot interact with each other. Because the vocabulary should meet the need of every agent role in the MAS, it is natural to define vocabulary at the end of requirement capture, after the roles and the quantity of each agent role has been clarified, and the informal description for each role's behavior has been harvested. After requirement capture, our modeling methodology can be applied in the MAS design stage, and the output from the design stage will be handled by the implementation phase.

Our modeling methodology can be briefly divided into the following five steps: (1) Acquire modeling requirements and the vocabulary from requirement capture phase; (2) Design the local plan for each agent; (3) Harvest resource requirements from local plans; (4) Design the coordinator; and (5) Generate the global plan. In Step 1, the modeling requirements and vocabulary are preliminary for model design, and they need to be organized into a specific format as we will see in Section 6.1. Local plan design (Step 2) generally requires human modeling experts. The last three steps are mainly automated, but we allow human experts to intervene in Step 4 for the purpose of providing modeling flexibility. Step 3 and Step 5 are completely automated. We further explain the design procedure in the rest of this section.

In the rest of this section, we use a simple multi-agent system as an example to demonstrate the modeling steps. The example system, called Simple Com, models commuting behavior of three agent-people, AP1,AP2 and $A P 3$. Each of the three agents describes options for a person's commuting behavior, and each agent has the same action model. The real difference in the modeling of the individual agents is in the path models, since in the path models, different agents contain different paths. Let AP1 be represented by the PERSON agent as was modeled in Section 4 and Section 5. For the purpose of integrating AP1 into our example, we use AP1's original action model (which is also now the action model for AP2 and AP3) but make minor revision to its path model to include variables in potential arcs. 


\subsection{Acquire modeling requirements and vocabulary}

To begin, we must gather the base modeling requirements, which includes the agent roles, the quantity of agents for each role, and the informal description of each role's behavior. The details of requirement capture are outside the scope of this paper since our focus is on the design stage. [BG04] provides a goal-oriented methodology that can aid in requirements capture.

Once we have the base requirements, we then must acquire a vocabulary. A complete vocabulary includes definitions to deal with all aspects of agent interactions, but the vocabulary in this paper is only concerned with conflict control related to resource sharing; therefore, we can focus on vocabulary elements needed to specify resources. We use CPN ML language [CPN06] to define a vocabulary, and further a communication language, which is based on the definition of vocabulary. Definition 6.1 defines a vocabulary, and Definition 6.2 defines a communication language. Each element in a vocabulary $V$ represents a name of an external resource that may be requested or released by agents in MAS designed, while a valid PI must be a multi-set over vocabulary $V$, i.e., a valid PI should be an element of the communication language. The semantics of the elements in a vocabulary are not interpreted by our modeling methodology, and they vary from application to application and must be agreed upon by the local plan designers during requirement capture. The adoption of CPN ML language in vocabulary and communication language definition provides the base for importing our models into CPN Tools for further analysis, since vocabulary and communication language can both be recognized and processed by CPN Tools.

Definition 6.1: A vocabulary $V$ is a color set specified by CPN ML language.

Definition 6.2: A communication language $C L$ is the set of all multi-sets on vocabulary $V$, i.e., $C L=V_{M S}$.

For example, the following statements written using CPN ML language defines color set CAR, which is a vocabulary. The communication language is the sets of multi-set on CAR, i.e., $C L=(C A R)_{M S}$.

colset ResType $=$ with car;

colset Color $=$ with red $\mid$ blue $\mid$ green;

colset $C A R=$ product ResType $*$ Color;

An element of CAR is a pair $(x, y)$, which defines one color token, and can be interpreted as name of a resource. For example, (car, red) is a single element of CAR; it is a colored token. While (car, red) intuitively means a red car, such a semantic is not interpreted by our modeling methodology and must be agreed upon by the agent designers using the vocabulary. The expression $2^{\prime}(\mathrm{car}, \mathrm{red})+3$ '(train, blue) is a CPN ML expression that evaluates to a multi-set on CAR; therefore, it is a valid PI (potential inscription) over the $C L=(C A R)_{M S}$. The color set CAR is used as the vocabulary for our example system, Simple Com.

We can also use variables in PIs. For example, $\mathrm{x}$ is a variable whose type is color set Color defined above. So $1\{$ car, $x\}+2\{$ train, blue $\}$ is also a valid PI. An expression such as $\{$ car, red, 1$\}$ would be an invalid PI on CL, although it intuitively means the same as 1 ' $\{$ car, red\}, i.e., a red car.

Note that our design procedure is not strictly limited to a vocabulary and communication language written in the CPN ML language - another notation could be used as long as the inscriptions written in the notation can be recognized and processed by all the agents and the coordinator. As mentioned before, in our current work, we adopt the CPN ML language to facilitate the use of existing CPN tools [CPN06]. 


\subsection{Design local plans and isolate resource requirements}

Local plan design is performed after requirement capture and vocabulary definition. Local plan design generally requires human modeling experts, so the local-plan design process cannot be completely automated.

For each agent, the local plan designer first develops an action model (the isolated version of local plan), and then creates alternative paths for each agent action in the CPN model. Replacing the action transition in CPN model with the paths for the agent action will generate a PCPN model, which is the interactive version of a local plan (path model). We already gave the guidelines for local plan design in Sections 4 and 5. Fig. 6.1 shows the graphical diagram for path models for the three agents $A P 1, A P 2$ and $A P 3$ in Simple Com. Each of the path models for $A P 1, A P 2$, and $A P 3$ includes only one path: (Move, 1 '(car, x), 0) for $A P 1$, (Move, 0,1 (car, blue)) for AP2, and (Move, 0, $1^{`}($ car, red)) for $A P 3$.

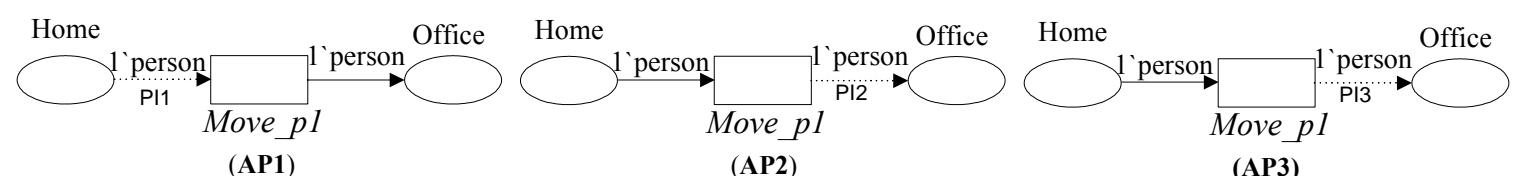

$\mathrm{PI} 11^{`}($ car, $x), \mathrm{PI} 2: 1^{`}$ (car, blue), $\mathrm{PI} 3: 1^{`}$ (car, red)

Fig. 6.1 Graphical Diagram for Agents in Simple Com

An advantage of our modeling methodology is its inherent support of independence of local plan designs. The coordinator can be unaware of modifications to a local plan, as long as the interaction interface of the path model remains intact. These interaction interfaces are specified in terms of the resource requirements carried by potential arcs. Consequently, only these resource requirements need to be transmitted to the coordinator designer. To facilitate this flow of information, we isolate these resource requirements in the form of a table for each path model; we call such a table an External Resource Table (ERT). Each entry in an ERT is a triple (t, type, PI), where $t$ is a path transition connected to a potential arc, type is request or release, and PI is the potential inscription carried by a potential arc. Intuitively, an entry ( $t$, type, PI) can be interpreted as meaning that the enabling of transition $t$ requires external resources specified by inscription PI (if type is request), or that the firing of transition $t$ should release external resources specified by inscription PI (if type is release).

For a path model, the number of potential inscriptions in the model equals to the number of entries in the corresponding ERT. ERT generation can be easily automated (See Appendix B1 for the algorithm details, and see Appendix B2 for comments and examples). The path model for AP1 in Fig 6.1 would generate an ERT for $A P 1$ containing the following single entry: (Move $p 1$, request, 1 ( $(\mathrm{car}, \mathrm{x})$ ). If variables are used in potential inscriptions and thereby harvested in ERTs, the definitions for those variables should also be included as part of resource requirements. For example, since variable $x$ is used by ERT entry (Move $p 1$, request, 1 (car, $\mathrm{x}$ )), the definition of $x$ (var x: Color ) needs to be provided to the coordinator designer as part of AP1's resource requirements.

\subsection{Design the coordinator}

To initiate the design of a coordinator module, the following information must first be accumulated: (1) the number of agents in our MAS, and the role of each agent; and (2) The resource requirements from every agent. If two agents belong to the same role, they have identical local plans, so the resource requirements for these two agents 
are the same. Local plans (both the isolated version and interactive version) are not used in the coordinator design. The major task in designing a coordinator model is to create transitions to represent agent actions and to connect these transitions with relevant resources, which are represented as CPN places. Through this procedure, a CPN model will be developed.

The coordinator design procedure can be divided into the following three steps: (1) global resource requirements coordination, (2) intermediate coordinator generation, and (3) complete coordinator generation. Step 1 accumulates the resource requirements provided by individual agents for the convenience of coordinator generation. Guided by the principle of "Separation of Concern," the coordinator generation process is split into two steps. An intermediate coordinator is first created by Step 2 to connect agent actions to resources. Such a coordinator is called an intermediate coordinator in the sense that it may contain unresolved variables. These variables will be resolved in Step 3 to develop the complete coordinator. If there is no unresolved variable in Step 2, the intermediate coordinator is equivalent to the complete coordinator. Both Step 1 and Step 2 can be automated. In Step 3, a "designer" must determine how variables will be resolved, and then an automated algorithm can be invoked to generate the complete coordinator. Here the designer would be an independent agent (human, software, etc.) capable of determining specific resources to be associated with specific agents/actions. Different variable resolution policies will lead to different complete coordinators. Therefore, the complete coordinator can be modified by revising variable resolution policies and then repeating Step 3 based on the intermediate coordinator obtained in Step 2. In this sense, our modeling methodology is flexible in coordinator generation. We now elaborate on the details of the three steps.

Step 1: Global resource requirements coordination. This step simply accumulates the External Resource Tables (ERTs), which were defined in Section 6.2, to provide a global view of resources that is independent of local plan details. (See Appendix $\mathrm{C} 1$ for the algorithm details and see Appendix $\mathrm{C} 2$ for comments and examples). For example, Fig. 6.2 identifies the ERT data for our Simple Com example. For this small example, there is only one entry for each of the three ERT tables. (Note that the name of an owner-agent (for example, AP1) is added as a prefix to the transition name in an ERT entry.)

ERT Table

Entries

\begin{tabular}{|l|l|}
\hline AP1.ERT & (AP1.Move_P1, request, 1'(car, $x$ )) \\
\hline AP2.ERT & (AP2.Move_P1, release, 1'(car, blue)) \\
\hline AP3.ERT & (AP3.Move_P1, release, 1'(car, red)) \\
\hline
\end{tabular}

Fig. 6.2 ERT Data for Simple Com

Step 2: Intermediate coordinator generation. The generation of the intermediate coordinator can be automated by generation of a special place node for each resource, the inclusion of transitions identified in the ERT data, and the use of arcs to model resource consumption and production (See Appendix D1 for the algorithm details and see Appendix D2 for comments and examples). For example, the ERT data identified in Fig. 6.2 can be used to generate the intermediate coordinator in Fig. 6.3. Note that there are no initial tokens for this intermediate coordinator, but it can be easily post-processed to add initial colored tokens to fit the modeling requirements. 


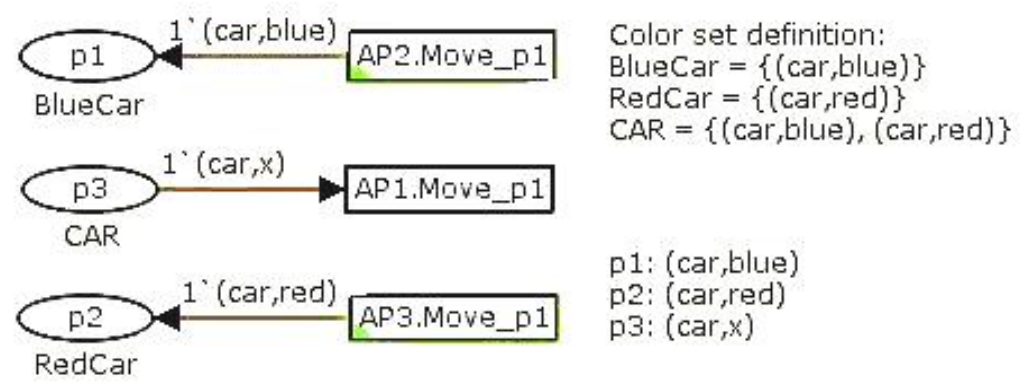

Fig. 6.3 Graphical Diagram Representation for an Intermediate Coordinator for Agents in Simple Com (variables unresolved)

Step 3: Complete coordinator generation. Since the intermediate coordinator may contain variables, we need to define binding policies (binding is defined in Section 3) to resolve the variables in the coordinator. Binding policies should decide which value(s) are allowed to be assigned to a specific variable at runtime. A designer can create binding policies to embed some important global-level attributes that can remain transparent to local plan designers. For example, in Simple Com if the supply of green cars is very limited, the coordinator designer can create binding policies to only allow blue and red cars to be allocated to AP1. We will see this binding policy "in action" shortly.

For the modeling methodology presented in this paper, a binding policy is defined as follows:

Definition 6.3 (Binding Policy): A binding policy is a pair (pre, post) where:

pre is a valid color token over a vocabulary and contains no variables;

post is a valid color token over a vocabulary and contains at least one variable.

pre and post should be color tokens over the same vocabulary.

As a simple example, consider the following binding policy for Simple Com: ( (car, red), (car, $x$ ) ), where

pre $=($ car, red $)$, and post $=($ car,$x)$. Binding policies are designed to specify which possible assignments are permitted in the coordinator. Thus, the binding policy ( (car, red), (car, $x)$ ) permits only token color red to be assigned to $x$.

After binding policies are defined, the intermediate coordinator can be modified according to the binding policies to form a complete coordinator. For each binding policy (pre, post), two transitions - we call them forward binding transition $(f t)$ and backward binding transition $(b t)$ - should be added into the intermediate coordinator. The following four arcs $a 1, a 2, a 3$, and $a 4$ should also be added into the coordinator for each binding policy: a1 = (Pre, $f t), a 2=(f t$, Post $), a 3=($ Post,$b t), a 4=(b t$, Pre $)$. Here Pre and Post refer to the corresponding resource place for token color pre and post in the coordinator, respectively. Inscriptions for all the four arcs are the same - 1 pre. It is natural to understand that different binding policies will lead to different coordinators, since binding policies affect how binding transitions will be added into the coordinator.

Returning to our example where there is a shortage of green cars, we might define two binding policies $(($ car,$r e d),($ car,$x))$ and ( (car, blue), (car, $x)$ ) for Simple Com (i.e., only allow red and blue cars). According to these two policies, four transitions and eight arcs are added into the coordinator. The resulting complete coordinator is shown in Fig. 6.4. Binding transitions $f t=t 13$ and $b t=t 31$ are added into the intermediate coordinator for binding 
policy ( (car, blue) , (car, $x)$ ). Four arcs are added to connect t13 and $\mathrm{t} 31$ to the coordinator, and the inscriptions for all the four arcs are the same: 1 ( (car, blue).

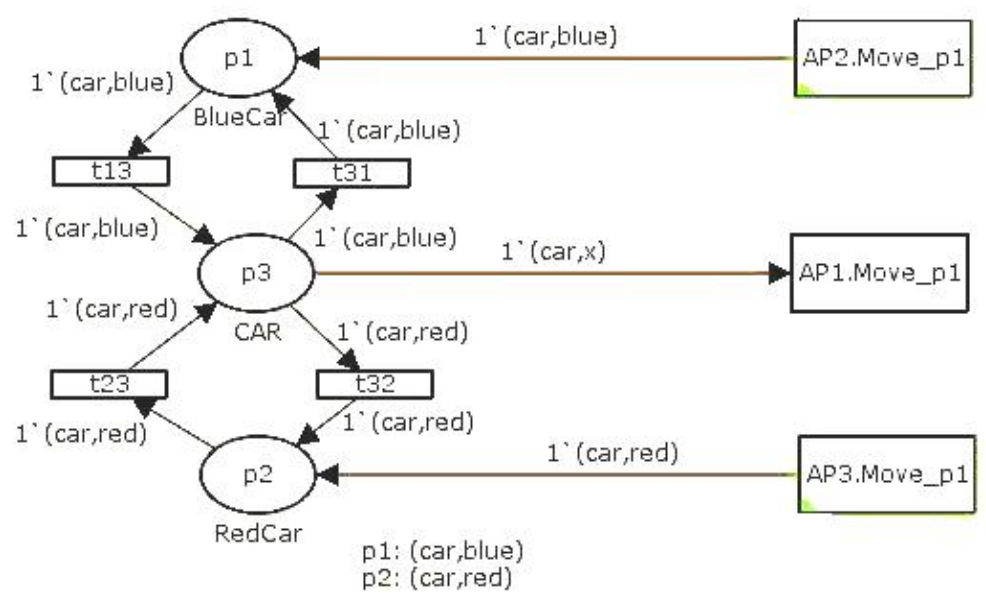

Fig. 6.4 Graphical Diagram for a Complete Coordinator for Simple Com (variable binding resolved)

\subsection{Generate the global plan}

Automatically concatenating local plans and a complete coordinator can generate the global plan for the MAS. The global plan is a regular Color Petri Net $G=\left(\Sigma_{g}, P_{g}, T_{g}, A_{g}, N_{g}, C_{g}, G_{g}, E_{g}, I_{g}\right)$. The global plan construction algorithm can be found in Appendix E1, with comments and examples in Appendix E2. Following the definition of global plan, we can concatenate path models of the three agents (Fig. 6.1) in Simple Com with the coordinator in Fig 6.4. The resulting global plan is shown in Fig. 6.5 as a graphical diagram.

Comparing Fig. 6.1 (path models), Fig. 6.4 (complete coordinator) and Fig. 6.5 (global plan), we can find that transition Move p1 in AP1's path model has been merged with transition AP1.Move p1 in the complete coordinator to create the transition AP1.Move $p 1$ in the global plan. Transition merging eliminates the potential inscriptions and finally casts the global plan as a CPN model. The global plan can then be analyzed by exploiting CPN analysis tools, such as CPN Tools [CPN06]. 


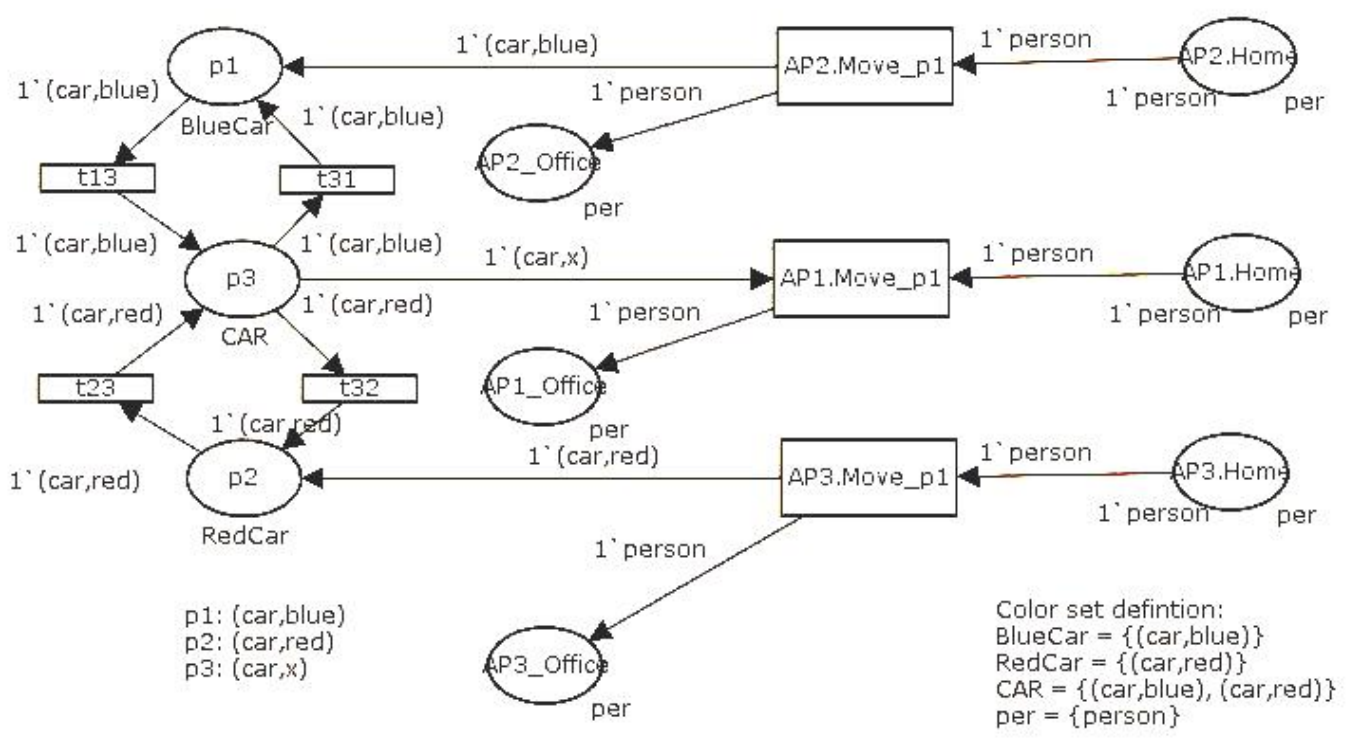

Fig. 6.5 The Graphical Diagram for Global plan for Simple Com

\section{Case Study}

\subsection{A simple scenario}

A family lives in the suburb of Chicago. The father needs to commute from home to office, and in the afternoon, the father may go to the park if the weather allows. In the case of bad weather, the father returns home directly from the office. The son is a college student, and he works part time every evening in a hospital to earn some money for his tuition. He sleeps in the morning, and goes to school every afternoon. The office, the school and the hospital are located in the city, while the park and father/son's home are in two different suburbs. There are three choices for transportation: car, train, or bus. However, not all options are available between two locations. For example, there is no train between the school and the hospital. The family has only one car; so if the father drives to work, the son cannot use the car until the father drives the car back home. The availability of trains and buses is not fully predictable, although it roughly follows some schedule.

Based on the scenario, we can develop a MAS model to describe the commuting behavior of the family, helping them to choose an appropriate approach at a specific moment. Here the car, trains and buses are described as external resources, and their availability changes dynamically in runtime. We assume that the weather conditions are not reliably predictable; this impacts the father's behavior, and the availability of the car.

\subsection{System design}

We design four agent roles in our system: father, son, train dispatcher and bus dispatcher. For this case study, we use one agent of each role and we adopt the color set RESOURCE, defined as follows, as the vocabulary.

colset ResType $=$ with car $\mid$ train $\mid$ bus;

colset Loc $=$ with home $\mid$ office $\mid$ park $\mid$ school $\mid$ hospital;

colset RESOURCE $=$ product ResType $*$ Loc; 
We followed the design procedure in Section 6 to generate a MAS system design. The detailed design procedure is omitted due to the lack of space, but Fig. 7.1 to Fig. 7.4 illustrates the path models for the four different agents. Inscriptions below arcs are potential inscriptions, while inscriptions above arcs are regular inscriptions.

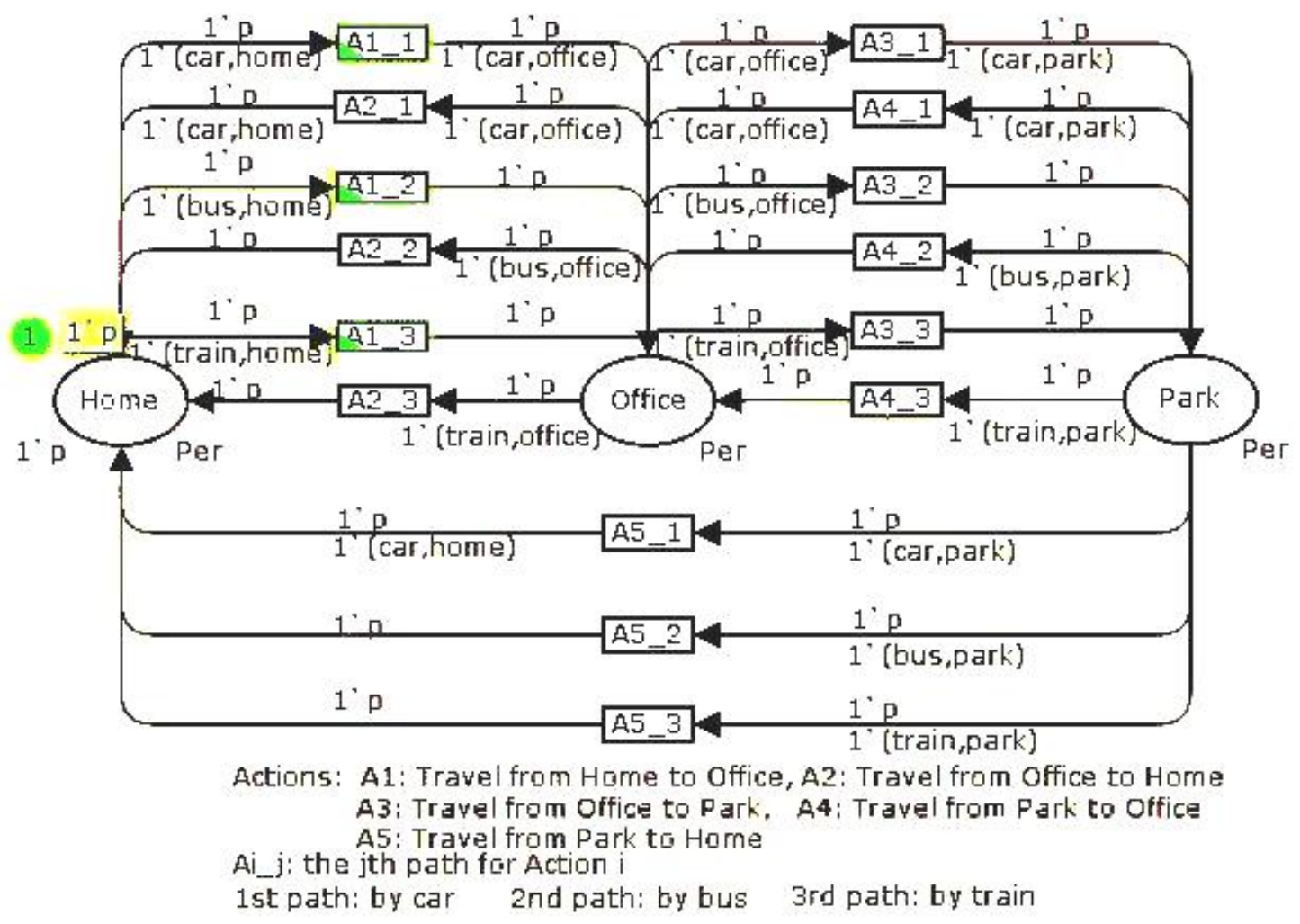

Fig. 7.1 The Father's Path Model

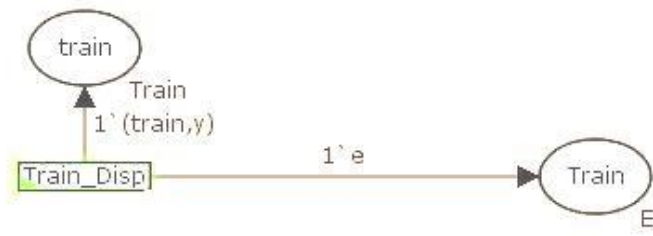

Fig. 7.2 The Train Dispatcher's Path Model

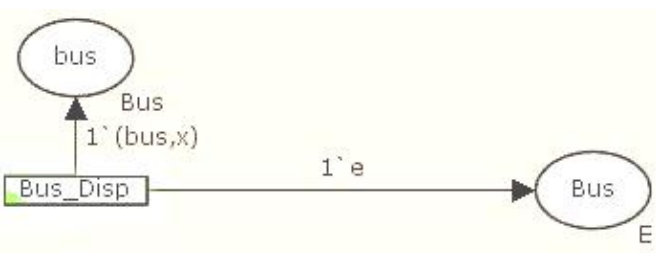

Fig. 7.3 The Bus Dispatcher's Path Model 


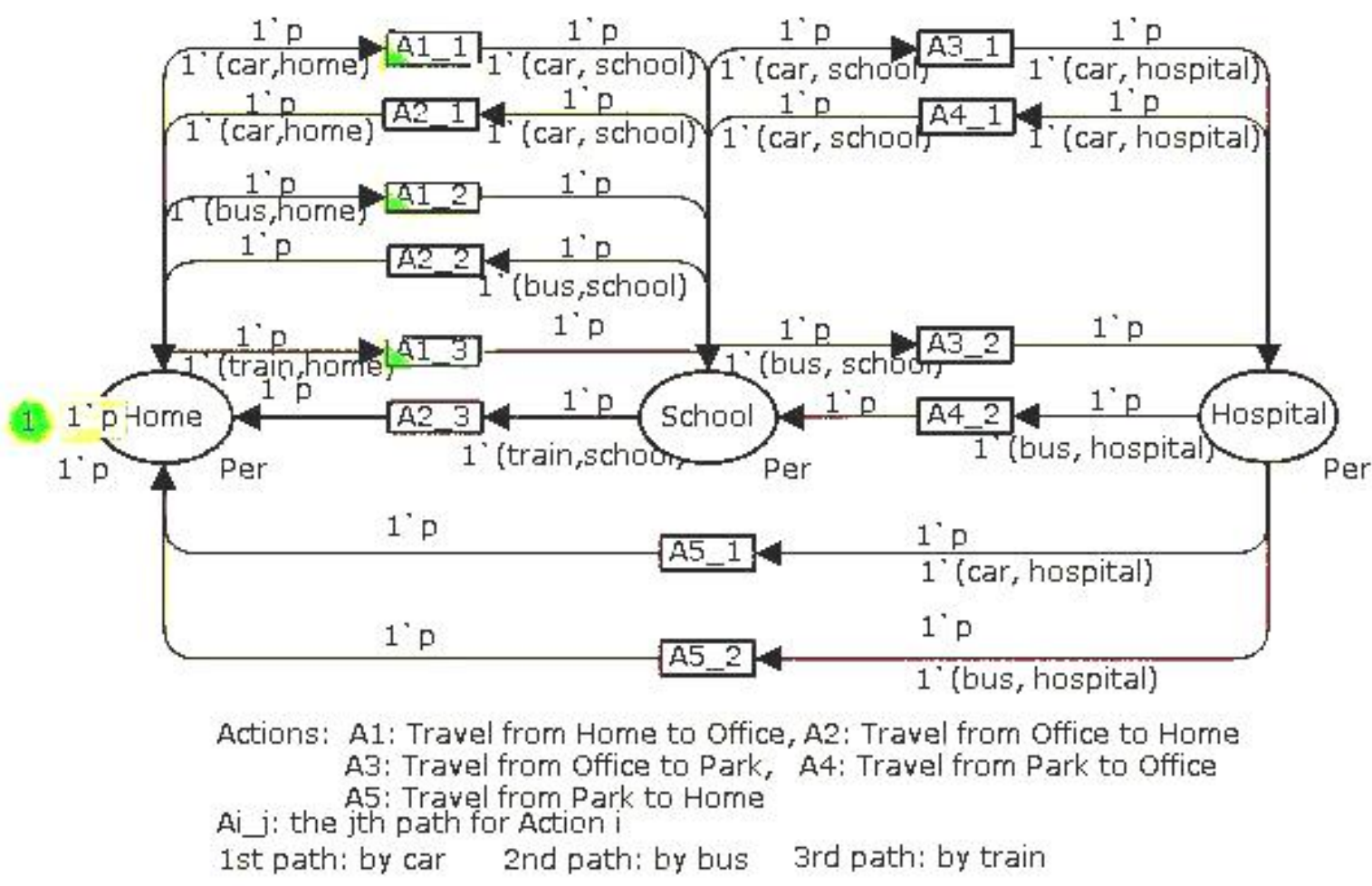

Fig. 7.4 The Son's Path Model

The train dispatcher's path model has only one path associated with the sole action (train) dispatch: dispatch a train to a location specified by variable $y$. Similarly, we get the bus dispatcher's path model. The bus dispatcher's path model also contains only one path associated with the sole action (bus) dispatch: dispatch a bus to a location specified by variable $x$. In both Fig. 7.2 and Fig. 7.3, 1 ' $e$ is the regular expression inherited from action models.

After local plans are developed, ERT tables are generated. We omit showing the ERT tables here due to space limitations. In the coordinator design step, we design binding policies to allow all the possible bindings for variable $x$ and $y$. The binding policies are listed as follows:

For variable $y$ :

1. ((train, home), (train, y)), 2. ((train, office), (train, y)),

3. ((train, park), (train, y)), 4. ((train, school), (train, y)).

For variable $x$ :

1. ((bus, home), (bus, x)), 2. ((bus, office), (bus, $x)$ ),

3. ((bus, park), (bus, $x))$, 4. ((bus, school), (bus, x)),

5. ((bus, hospital), (bus, $x))$.

Since the coordinator is quite large, we only show parts as an example. Fig. 7.5 shows the transitions connected to all type of cars. In Fig 7.5, no binding transition exists because no variable related to car exist. In contrast, Fig. 7.6 shows transitions connected to the resource train. There are eight binding transitions in Fig. 7.6, 
corresponding to the four binding policies for variable $y$ in token (train, y). From Fig. 7.5 and Fig. 7.6, we can clearly capture the interactions surrounding a specific resource without concern for other parts of the MAS model.

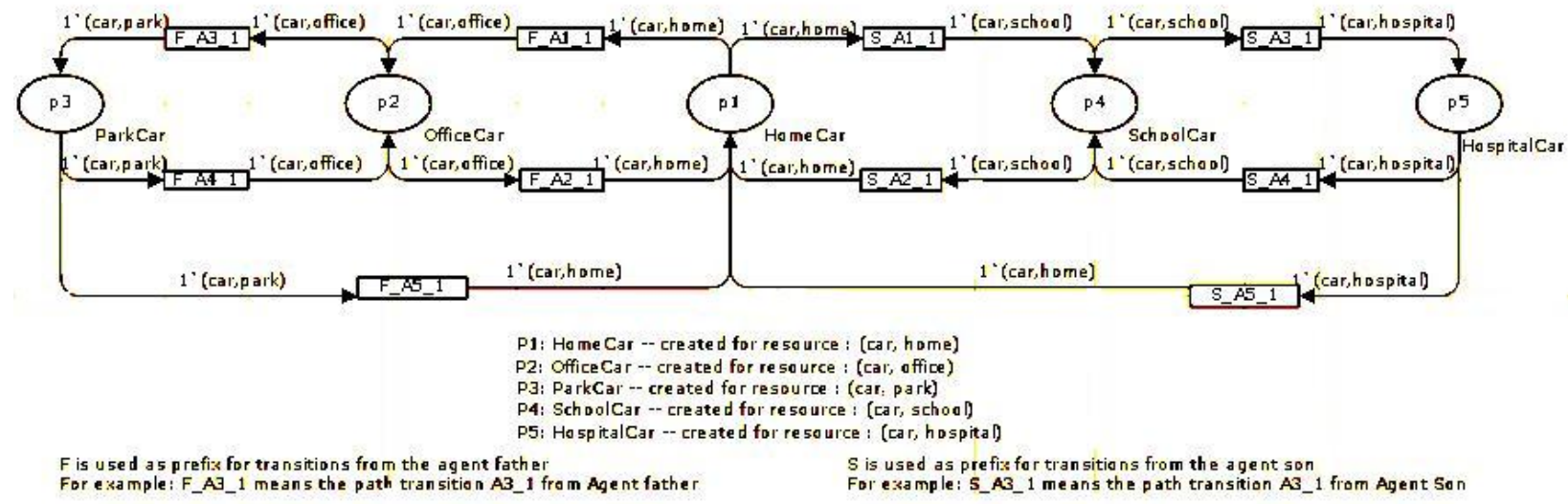

Fig. 7.5 Part of the Coordinator Related to Cars at Different Locations

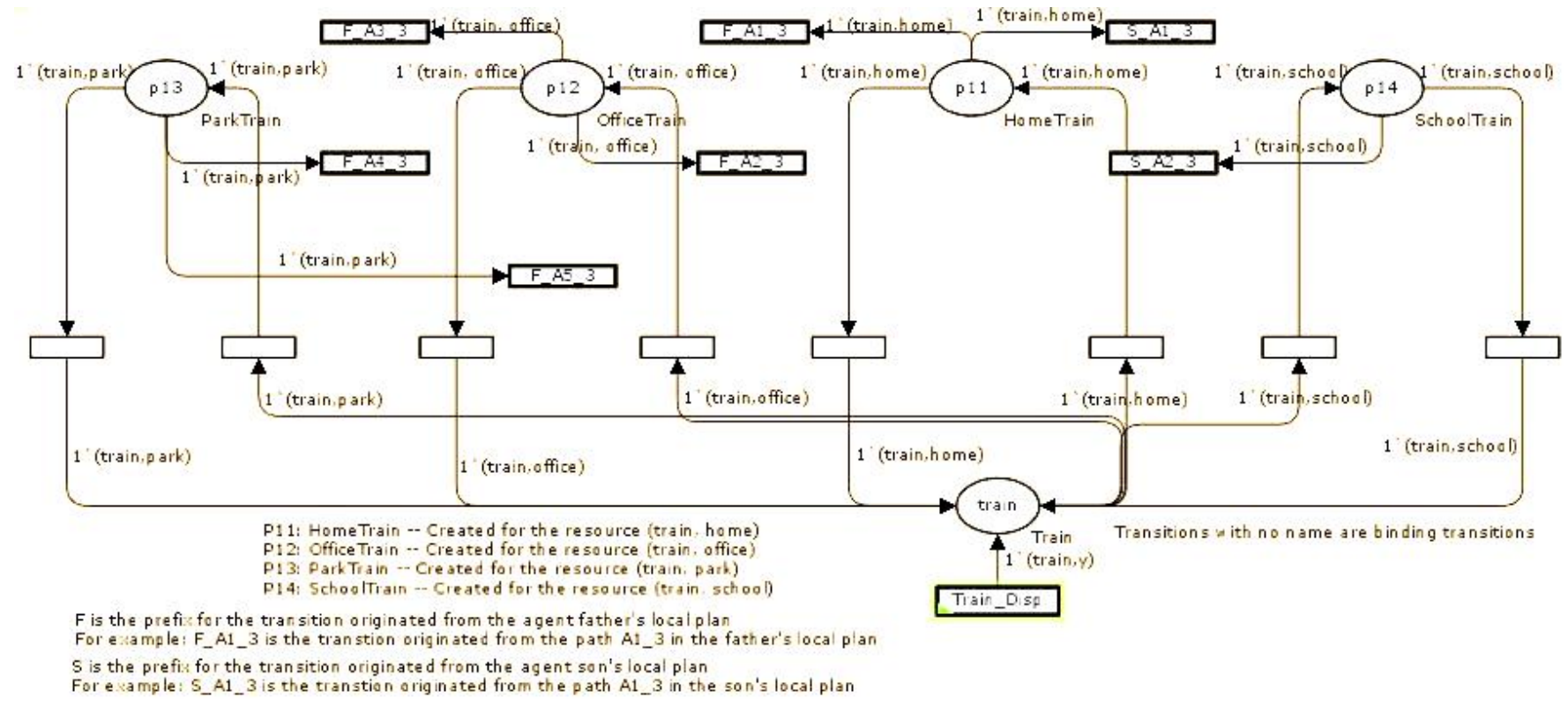

Fig. 7.6 Part of the Coordinator Related to Trains at Different Locations

Finally, generating the global plan means merging the transitions from local plans and the transitions from the coordinator. Due to the large size of the global plan, substitution transitions are adopted to present the global plan. Substitution transitions are supported by CPN tools [CPN 06] to create a large CPN diagram in multiple layers of details. The partial global plan (only graphical diagram) presented in Fig 7.7 has two layers. The upper half of Fig 7.7 is a super-page, presenting a broad overview for the global plan (only the part related to resource train). All the path transitions in Fig 7.6 are cast as substitution transitions in the super page of Fig. 7.7 (shown with thicker borders). Each substitution transition in the super-page is matched to a sub-page, shown in the lower half of Fig. 7.7. 


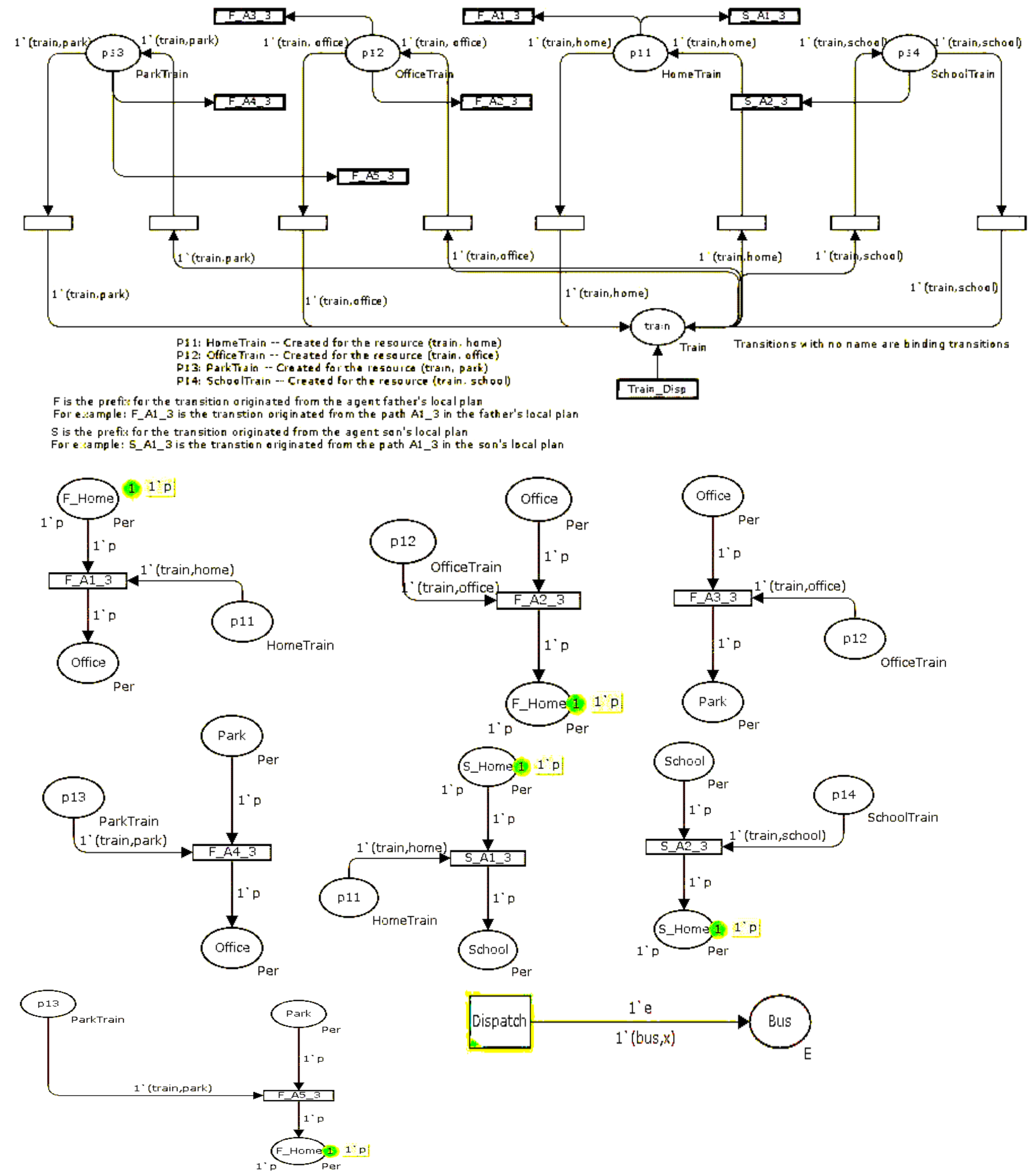

Fig. 7.7 Part of the Global Plan Related to Trains at Different Locations 


\subsection{Preliminary simulation and analysis}

One of the advantages of building net models is that the analysis of the models can be supported by existing, mature analysis tools. Although the primary focus of this paper is with the modeling methodology for MASs, we present some preliminary analysis results to illustrate the viable use of Petri net based analysis for our domain of interest.

We imported the global plan developed in Section 6.1 into CPN Tools for analysis. The global plan is simulated to study the run-time behavior of the MAS, which will be developed based on the global plan. During the simulation, if multiple transitions are enabled at a specific step, CPN Tools randomly select an enabled transition to fire. Through analyzing the simulation results, we inspected one type of possible conflicts in the MAS: the father and the son use the same travel resource (car, train, or bus) to leave home, when the same travel resource cannot be available to both of them. (The father leaves home for office, and the son leaves home for school). The triggering event for this conflict can be represented as: both $F_{-}$Home and $S_{-}$Home (See Fig. 7.7) have tokens at a simulation step. To resolve this conflict, the global plan needs to guarantee that the related actions - action A1 of the father and action A1 of the son - are taken through available paths, while the availability of a path is determined by the availability of resources (car, train and bus). An example for the failure in avoiding such a conflict can be described as follows: if there is only bus and train available, the father takes the car to leave home. The Petri net token-driven mechanism guarantees the alleged failure above won't occur, as we will see in the simulation results.

CPN Tools provide a set of mechanisms to facilitate the analysis of Petri net models, and these mechanisms can tailor the simulation traces for various analysis purposes. In CPN Tools, a monitor is a mechanism that is used to observe, inspect, control, or modify a simulation of a colored Petri net. Monitors can inspect both the markings of places and the occurring binding elements during a simulation, and they can take appropriate actions based on the observations. We used two monitors to control the generation of the simulation traces in our experiments. One monitor inspected the marking of place $F_{-}$Home (originated from the Home Place in the father's local plan), the marking of place $S_{-}$Home (originated from the Home Place in the son's local plan) and the availability of paths at each simulation step. The markings of $F_{-}$Home and $S_{-}$Home are used to detect conflicts. Another monitor recorded the firing of path transitions during the simulation. After each run of the simulation, the trace files are postprocessed to abstract and interpret the information contains in trace files. As a result, a simulation report will be generated. A partial simulation report is shown as follows:

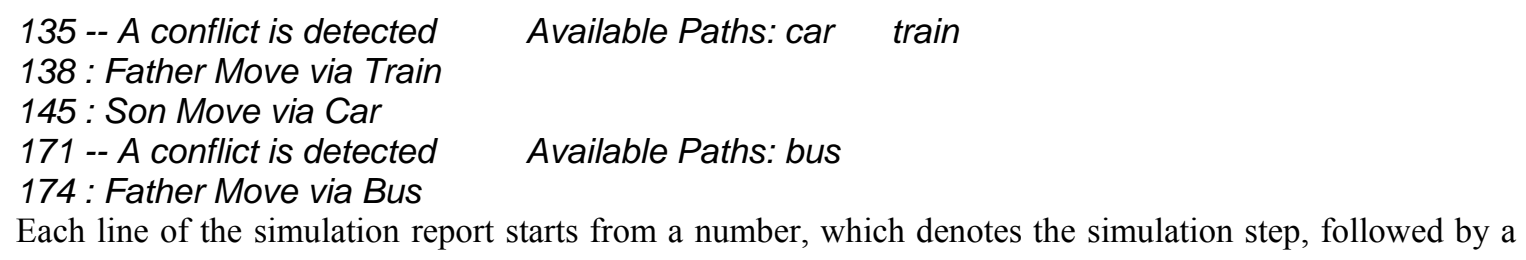
record section. A record section can either report the detection of a conflict with the available paths denoted by the related resource at the step of conflict, or interpret the path transition fired in the step to show the action taken to avoid a conflict. For instance, from the sample report above, we know that at step 135, a conflict is detected, and the available paths are: (1) by car, and (2) by train. At step 138, a path transition is taken to resolve the conflict, which means the father takes the train to move (Transition F_A1_3 of Fig. 7.7 is fired). Five Simulation reports were 
generated, each of which contained the information abstracted from a simulation run of 10000 steps. Inspection of these simulation reports by a simple algorithm (the details of the algorithm is omitted due to the concern of space) confirms that all the conflicts are resolved by taking an available path and there is no failure. This result confirms that our modeling methodology can support independent individual agent development, and resolve the run-time conflicts without dynamically computing a path.

Also, through analyzing the simulation reports, we can predict how different resources are used in the MAS, and such prediction is useful to revise the design of the MAS to achieve better resource utilization, as we have claimed to be one advantage of the dynamic avoidance approach. Fig 7.8 shows the number of detected conflicts in each simulation report and the paths taken to resolve each conflict. (Note that for report 2 and 3, there is one more conflict detected than those resolved, due to the fact that the simulation is cut off at 10000 steps, so that the resolution of the last detected conflict is not included in the simulation report. Also, the corresponding path transition for each path is shown in the parenthesis). We can see from Fig. 7.8 that the bus and the train are more frequently used to resolve the conflicts than the car. The reason is that the randomness in the transition selection during the simulation made the path transitions associated to the bus and the path transition associated to the train more likely to be available than the path transition associated with the car. Based on this finding, the global plan can be revised to achieve better resource utilization, for instance, more car tokens are added into the coordinator to relief the pressure on the bus and the train.

\begin{tabular}{|l|l|l|l|l|l|l|}
\hline \multicolumn{2}{|l|}{ Report Index } & 1 & 2 & 3 & 4 & 5 \\
\hline Conflicts & 246 & 215 & 227 & 283 & 236 \\
\hline \multirow{3}{*}{ Path: } & Father Takes Car (F_A1_1) & 11 & 18 & 15 & 23 & 17 \\
\cline { 2 - 7 } & Father Takes Bus (F_A1_2) & 45 & 37 & 48 & 56 & 47 \\
\cline { 2 - 7 } & Father Takes Train (F_A1_3) & 66 & 58 & 60 & 65 & 64 \\
\cline { 2 - 7 } & Son Takes Car (S_A1_1) & 9 & 13 & 5 & 27 & 13 \\
\cline { 2 - 7 } & Son Takes Bus (S_A1_2) & 55 & 39 & 39 & 56 & 39 \\
\cline { 2 - 7 } & Son Takes Train (S_A1_3) & 60 & 49 & 59 & 56 & 56 \\
\hline
\end{tabular}

Fig. 7.8 Detected and Resolved Conflicts in Each Simulation Report (path transitions are shown in parenthesis)

\section{Conclusion and future research}

Briefly speaking, the potential arc concept expands the modeling power of CPN to distinguish the representation of potential conflicts from real conflicts in MAS design. Based on potential arc concept, we develop a new MAS modeling methodology. The algorithms in our modeling methodology provide the base for modeling automation. Further research includes the integration of agent reasoning mechanism to our modeling methodology to provide more comprehensive agent models. Also, research will be continued on both simulation based and model checking based model analysis. 


\section{References}

[BL99] K. S. Barber, T. H. Li , A. Goel and C. E. Martin, "Conflict Representation and Classification in a Domain Independent Conflict Management Framework," Proceedings of the Third International Conference on Autonomous Agents, 1999.

[BL01] K. S. Barber, T. H. Liu and S. Ramaswamy, "Conflict Detection During Plan-Integration for Multi-Agent Systems," IEEE Transactions on Systems, Man, and Cybernetics, Vol. 31, Number 4, August 2001.

[BG04] P. Bresciani, P.Giorgini, F. Giunchiglia, J. Mylopoulos and A. Perini, "TROPOS: An Agent-Oriented Software Development Methodology," Journal of Autonomous Agents and Multi-Agent Systems, 8(3):203-236, 2004 [CPN06] CPN ML language, From CPN Tools (Version 2.0) help file, 2006.

[CY03] L. M. Cysneiros and E. Yu, "Requirements Engineering for Large-Scale Multi-agent Systems," Lecture Notes in Computer Science. Vol. 2603, pp. 39-56, Springer 2003.

[DW01] S. A. Deloach, M. Wood and C. Sparkman, "Multi-agent System Engineering," International Journal of Software Engineering and Knowledge Engineering, 11(3): 231-258, April, 2001.

[FG05] G. Fortino, A. Garro and W. Russo, "An Integrated Approach for the Development and Validation of Multiagent Systems," International Journal of Computer Systems Science and Engineering. Vol. 20, Number 4, July 2005. [GH97] S.Green, L. Hurst, B. Nangle, P. Cunningham, F. Somers and R. Evans, "Software Agents: A Review," Intelligent Agent Group(IAG) report TCD-CS-1997-06, Trinity College Dublin May 1997.

[H95] T. Holvoet, “Agents and Petri Nets,” Petri Net Newsletters, Number 49, 1995.

[HB04] C. Hanachi and C. S. Blanc, "Protocol Moderators as Active Middle-Agents in Multi-Agent Systems," Autonomous Agents and Multi-Agent Systems. Vol. 8, pp. 131-164, no. 2, 2004.

[HM04] R. S. Herrera and E. L. Mellado, "Modular and Hierarchical Modeling of Interactive Mobile Agents" Systems, Man and Cybernetics, 2004 IEEE International Conference, Vol. 2, pp. 1740 - 1745, 10-13 Oct. 2004.

[J96] N.R. Jennings, "Coordination Techniques for Distributed Artificial Intelligence," in Foundation of Distributed Artificial Intelligence, Sixth-Generation Computer Technology Series, G. M. P. O'Hare and N. R. Jennings, Eds, New York: Wiley, 1996, pp. 187-210.

[J97] K. Jensen, “Coloured Petri Nets: Basic Concepts, Analysis Methods and Practical Use," Springer Verlag, 2nd corrected printing 1997.

[J01] N. R. Jennings, “An Agent-Based Approach for Building Complex eSoftware Systems," Communications of $A C M$. Vol. 44, no. 4, April 2001, pp. 35-41.

[JSM98] N. R. Jennings, K. Sycara, and M. Wooldridge, "A Roadmap of Agent Research and Development." In: Autonomous Agents and Multi-Agent Systems Journal, Volume 1, Issue 1, pages 7-38, 1998.

[JS06] J. Lian and S. M. Shatz, "Potential Arc: A Modeling Mechanism for Conflict Control in Multi-Agent Systems," Proceedings of the $4^{\text {th }}$ Symposium on Design, Analysis, and Simulation of Distributed Systems (DASD06), Huntsville, Alabama, April 2006, pp. 467 - 474.

[KG96] D. Kinny, M. Georgeff and A. Rao, "A Methodology and Modeling Technique for Systems of BDI Agents," Proceedings of the Seventh European Workshop on Modeling Autonomous Agents in a Multi-Agent World, 1996. 
[KG97] D. Kinny and M. P. Georgeff, "Modeling and Design of Multi-Agent Systems," Proceeding of the 4th International Workshop on Agent Theories, Architectures, and Language (ATAL-97), 1997, pp. 1-20.

[LT05] I. Loutchko and F. Teuteberg, "An Agent-based Electronic Job Marketplace: Conceptual Foundations and FuzzyMAN Prototype," International Journal of Computer Systems Science \& Engineering, 2005.

[LR78] L.H. Landweber and E.L.Robertson, "Properties of Conflict Free and Persistent Petri Nets," Journal of the $A C M$, Vol. 25, No. 3, pp. 352-364, July 1978.

[M89] T. Murata, "Petri Nets: Properties, Analysis and Application," Proceeding of the IEEE, Vol. 77, Number 4, April 1989, pp. 541-580.

[MP95] J. J. Moder, C.R. Phillips, and E. W. Davis, Project Management with CPM,PERT and Precedence Diagramming. Blitz Publishing Company; 3rd edition (February 1995).

[MN91] T. Murata, P.C. Nelson and J.Yim, "Predicate-Transition Net Model for Multiple Agent Planning," Information Science, Vol. 57/58, pp. 361-384, 1991.

[MT96] P. Moraïtis and A. Tsoukiàs, "A Multi-criteria Approach for Distributed Planning and Conflict Resolution for Multi-Agent Systems," Proceeding of International Conference on Multi Agent Systems, 1996.

[MW97] D. Moldt and F. Wienberg, "Multi-Agent-Systems Based on Coloured Petri Nets." Proceeding of 18th International Conference on Application and Theory of Petri Nets, 1997.

[RH03] S. Resmerita and M. Heymann, "Conflict Resolution in Multi-Agent Systems," Proceeding of 42nd IEEE Conference on Decision and Control, 2003.

[RHM03] S. Resmerita, M. Heymann and G. Meyer, "A framework for conflict resolution in air traffic management," Proceeding of 42nd IEEE Conference on Decision and Control, Vol. 2, pp. 2035 - 2040, Dec. 2003

[S93] Y. Shoham, “Agent-Oriented Programming,” Artificial Intelligence, Vol. 60, pp. 51-92, 1993.

[S94] J. A. Sillince, "Multi-agent Conflict Resolution: a Computational Framework for an Intelligent Argumentation Program," Knowledge Based Systems, Vol. 7, Number 2, June 1994.

[WJ00] M. Wooldridge, N. R. Jennings and D. Kinny, "The Gaia Methodology for Agent-oriented Analysis and Design," Journal of Autonomous Agents and Multi-Agent Systems, 3(3):285-312, 2000.

[WH02] D. Weyns and T. Holvoet, "A Colored Petri Net for a Multi-Agent Application," Proceeding of Modeling of Object, Components and Agents, MOCA'02, Aarhus, Denmark August 26-27, pp.121-140, 2002.

[WH04] D. Weyns and T. Holvoet, "A Colored Petri Net for Regional Synchronization in Situated Multi-Agent Systems," Proceeding of First International Workshop on Petri Nets and Coordination (PNC), Bologna, Italy, June 21-26, 2004.

[WR06] T. Wagner, A. Raja, and V. Lesser "Modeling Uncertainty and its Implications to Sophisticated Control in TAEMS Agents," Autonomous Agents and Multi-Agent Systems, Vol. 13, No. 3, pp. 235 - 292, 2006.

[XS03] H. Xu and S. M. Shatz, “A Framework for Model-Based Design of Agent-Oriented Software," IEEE Transactions on Software Engineering, Vol. 29, No. 1, January 2003, pp. 15-30.

[XV03] D. Xu, R. A. Volz, T. R. Ioerger and J. Yen, "Modeling and Analyzing Multi-agent Behaviors Using Predicate/transition Nets," International Journal of Software Engineering, Vol. 13, No. 1, 2003, pp 103-124. 


\section{Appendix:}

This appendix gives the details for five algorithms used in the modeling methodology discussed in Section 6. We use the sample MAS - Simple Com (introduced at the beginning of Section 6) - as an example to illustrate the algorithms in this appendix. Two color sets are defined here by CPN ML language:

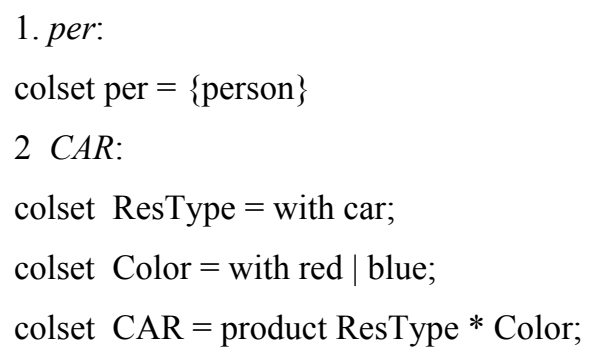

\section{Appendix A1: Path Model Generation Algorithm}

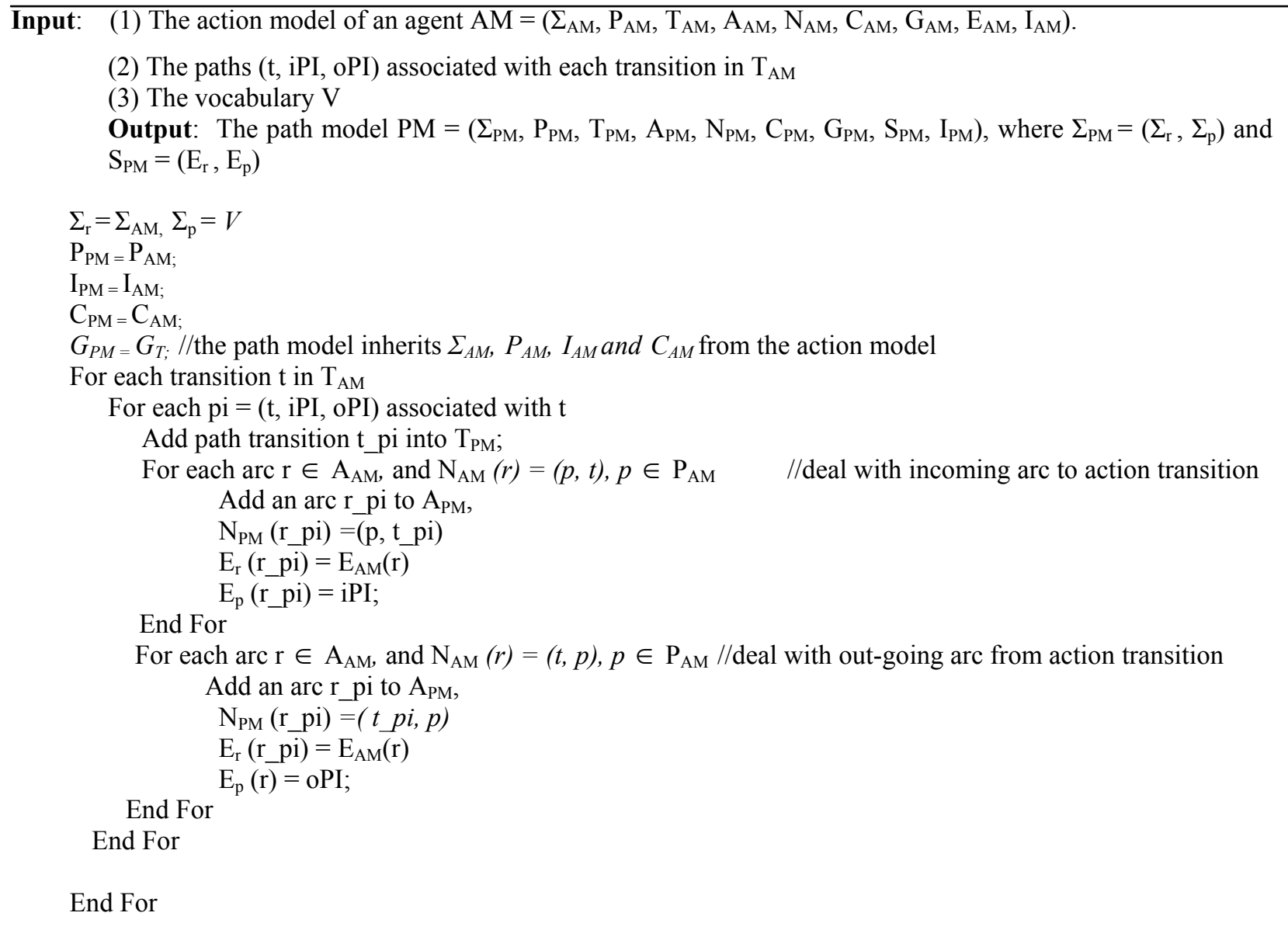

\section{Appendix A2: Comments and Examples for Path Model Generation Algorithm}

The path model generation algorithm takes an action model and paths for each agent action as input, and the corresponding path model as an output. The kernel idea in this algorithm is to replace each action transition in a 
path $(t, i P I, o P I)$ with the path transitions of the corresponding action. And the arcs connected to an action transition in the action model will now be connected to each of the corresponding path transitions in the path model. If multiple paths are associated with an action transition, the same number of path transitions will also be generated to form a path model, one path transition for each path. The potential arc inherits the regular inscription from the action model, and acquires an extra transition provided by the path. For the action model of $A P 1$ in Fig 4.1 of Section 4, if we have only one path $p 1=($ Move, 1 ( $(c a r, x), 0)$ for the sole action transition Move, after applying the path model generation algorithm above, we got the resulting path model with textual notation in Fig 1(1) in this appendix. In Fig. 1(1), path transition Move pl is generated for action transition Move. Because the path (Move, I (car, x), 0) needs the external resource 1 (car, $x)$, the arc $\left(r 1 \_p 1\right.$, Move $\left.\_1\right)$ is designed as a potential arc with the potential inscription $l$ ' $c a r$. It is worth noting that $p 1$ contains variable $x$, for the reason that we don't want to specify the color of the car. We also design path model of AP2 to contain a path $p 1=$ (Move p1,0, 1 (car, blue)) and path model of AP3 to contain a path $p 1=($ Move $p 1,0,1$ ( $($ car, red $)$ ). Path models for AP2 and AP3 are expressed as textual notation in Fig 1(2) and Fig 1 (3) respectively. Also, Fig. 6.1 shows the graphic diagram of the three path models in Simple Comm.

$\mathrm{PM}=\left(\Sigma_{\mathrm{PM}}, \mathrm{P}_{\mathrm{PM}}, \mathrm{T}_{\mathrm{PM}}, \mathrm{A}_{\mathrm{PM}}, \mathrm{N}_{\mathrm{PM}}, \mathrm{C}_{\mathrm{PM}}, \mathrm{G}_{\mathrm{PM}}, \mathrm{S}_{\mathrm{PM}}, \mathrm{I}\right)$, where:

$\Sigma_{\mathrm{PM}}=\left(\Sigma_{\mathrm{r}}, \Sigma_{\mathrm{p}}\right)$, where

$\Sigma_{\mathrm{r}}=\{$ per $\}$.

$\Sigma_{\mathrm{p}}=\{C A R\}$

$\mathrm{P}_{\mathrm{PM}}=\mathrm{P}_{\mathrm{AM}}=\{$ Home, Office $\}$

$\mathrm{T}_{\mathrm{PM}}=\{$ Move $p 1\}$.

$A_{\mathrm{PM}}=\{r 1 p 1, r 2 p 1\}$.

$N_{\mathrm{PM}}=\left\{N\left(r 1 \_p 1\right)=(\right.$ Home, Move $p 1), N\left(r 2 \_1\right)=\left(\right.$ Move $\_$l 1 , Office $\left.)\right\}$.

$\mathrm{C}_{\mathrm{PM}}=\mathrm{C}_{\mathrm{AM}}=(C($ Home $)=$ per, $C($ Office $)=$ per $)$.

$G_{\mathrm{PM}}=G_{T}$

$S_{\mathrm{PM}}=\left(E_{r}, E_{p}\right)$ where:

$E_{r}=\left\{E_{r}(r 1 p 1)=1\right.$ person, $E_{r}(r 2 p 1)=1$ 'person $\}$.

$E_{r}=\left\{E_{p}(r 1 p 1)=1\right.$ (car, $\left.\left.x\right), E_{p}(r 2 p 1)=0\right\}$.

$\mathrm{I}_{\mathrm{PM}}=\mathrm{I}_{\mathrm{AM}}=\{$ I(Home $)=1$ 'person, $\mathrm{I}($ Office $\left.)=0\right\}$.

(1) AP1 (revised)

$\mathrm{PM}=\left(\Sigma_{\mathrm{PM}}, \mathrm{P}_{\mathrm{PM}}, \mathrm{T}_{\mathrm{PM}}, \mathrm{A}_{\mathrm{PM}}, \mathrm{N}_{\mathrm{PM}}, \mathrm{C}_{\mathrm{PM}}, \mathrm{G}_{\mathrm{PM}}, \mathrm{S}_{\mathrm{PM}}, \mathrm{I}\right)$, where:

$\Sigma_{\mathrm{PM}}=\left(\Sigma_{\mathrm{r}}, \Sigma_{\mathrm{p}}\right)$, where

$\Sigma_{\mathrm{r}}=\{$ per $\}$.

$\Sigma_{\mathrm{p}}=\{C A R\}$

$\mathrm{P}_{\mathrm{PM}}=\mathrm{P}_{\mathrm{AM}}=\{$ Home, Office $\}$

$\mathrm{T}_{\mathrm{PM}}=\{$ Move $p 1\}$.

$A_{\mathrm{PM}}=\{r 1 p 1, r 2 p 1\}$.

$N_{\mathrm{PM}}=\left\{N\left(r 1 \_\right.\right.$1) $=\left(\right.$Home, Move $\left.\_1\right), N\left(r 2 \_\right.$1) $=\left(\right.$Move $\_1$, Office $\left.)\right\}$.

$\mathrm{C}_{\mathrm{PM}}=\mathrm{C}_{\mathrm{AM}}=(C($ Home $)=$ per, $C($ Office $)=$ per $)$.

$G_{\mathrm{PM}}=G_{T}$

$S_{\mathrm{PM}}=\left(E_{r}, E_{p}\right)$ where:

$E_{r}=\left\{E_{r}(r 1 p 1)=1\right.$ person, $E_{r}(r 2 p 1)=1$ 'person $\}$.

$E_{p}=\left\{E_{p}\left(r 1 \_1\right)=0, E_{p}\left(r 2 \_1\right)=1(\right.$ car, blue $\left.)\right\}$.

$\mathrm{I}_{\mathrm{PM}}=\mathrm{I}_{\mathrm{AM}}=\{$ I(Home $)=1$ 'person, $\mathrm{I}($ Office $\left.)=0\right\}$. 
$\mathrm{PM}=\left(\Sigma_{\mathrm{PM}}, \mathrm{P}_{\mathrm{PM}}, \mathrm{T}_{\mathrm{PM}}, \mathrm{A}_{\mathrm{PM}}, \mathrm{N}_{\mathrm{PM}}, \mathrm{C}_{\mathrm{PM}}, \mathrm{G}_{\mathrm{PM}}, \mathrm{S}_{\mathrm{PM}}, \mathrm{I}\right)$, where:

$\Sigma_{\mathrm{PM}}=\left(\Sigma_{\mathrm{r}}, \Sigma_{\mathrm{p}}\right)$, where

$\Sigma_{\mathrm{r}}=\{$ per $\}$.

$\Sigma_{\mathrm{p}}=\{C A R\}$

$\mathrm{P}_{\mathrm{PM}}=\mathrm{P}_{\mathrm{AM}}=\{$ Home, Office $\}$

$\mathrm{T}_{\mathrm{PM}}=\left\{\right.$ Move $\_$p $\}$.

$A_{\mathrm{PM}}=\left\{r 1 \_p 1, r 2 \_p 1\right\}$.

$N_{\mathrm{PM}}=\left\{N\left(r 1 \_1\right)=(\right.$ Home, Move $p 1), N\left(r 2 \_1\right)=\left(\right.$ Move $\_1$, Office $\left.)\right\}$.

$\mathrm{C}_{\mathrm{PM}}=\mathrm{C}_{\mathrm{AM}}=(C($ Home $)=$ per, $C($ Office $)=$ per $)$.

$G_{\mathrm{PM}}=G_{T}$

$S_{\mathrm{PM}}=\left(E_{r}, E_{p}\right)$ where:

$E_{r}=\left\{E_{r}(r 1 p 1)=1\right.$ person, $E_{r}(r 2 p 1)=1$ 'person $\}$.

$E_{r}=\left\{E_{p}(r 1 p 1)=0, E_{p}(r 2 p 1)=1(\right.$ car, red $\left.)\right\}$.

$\mathrm{I}_{\mathrm{PM}}=\mathrm{I}_{\mathrm{AM}}=\{\mathrm{I}($ Home $)=1$ 'person, $\mathrm{I}($ Office $)=0\}$.

(3) AP3

Fig. 1. Textual Notations for Path Models for Agents in Simple Com

\section{Appendix B1: External Resource Table (ERT) Generation Algorithm}

Input: A path model $\mathrm{PM}=(\Sigma, \mathrm{P}, \mathrm{T}, \mathrm{A}, \mathrm{N}, \mathrm{C}, \mathrm{G}, \mathrm{S}, \mathrm{I})$ of an agent $\mathrm{AP}$, where $\Sigma=\left(\Sigma_{\mathrm{r}}, \Sigma_{\mathrm{p}}\right)$ and $\mathrm{S}=\left(\mathrm{E}_{\mathrm{r}}, \mathrm{E}_{\mathrm{p}}\right)$

Output: An ERT of an agent

Create an empty ERT with no entry

For each element $\mathrm{r}$ in $A$

If $\left(\mathrm{E}_{\mathrm{p}}(\mathrm{r}) \neq 0\right) \quad / /$ only handle potential arc

If $\left(N_{\mathrm{PM}}(\mathrm{r})=(p, t) \in \mathrm{P}_{\mathrm{PM}} \times T_{\mathrm{PM}}\right) / / \mathrm{r}$ is an incoming arc

Add a triple $\left(A P \_t\right.$, request, $\left.\mathrm{E}_{\mathrm{p}}(\mathrm{r})\right)$ to ERT

if $\left(N_{\mathrm{PM}}(\mathrm{r})=(t, p) \in \mathrm{T}_{\mathrm{PM}} \times P_{\mathrm{PM}}\right) / / \mathrm{r}$ is an outgoing arc

End IF

Add a triple $\left(A P_{-} t\right.$, release, $\left.E_{p}(r)\right)$ to ERT

End For

\section{Appendix B2: Comments and Examples for External Resource Table (ERT) Generation}

The major purpose of ERT generation algorithm is to re-organize information carried by potential inscriptions, so that ERTs can be easily processed during the coordinator design.

The path model for AP1 in Fig 1 (1) has only two arcs $\left(r 1 \_p 1\right.$, and $\left.r 2 \_1\right)$. Since $\mathrm{E}_{\mathrm{p}}\left(r_{2} \_p 1\right)=0$, no corresponding ERT entry for $r 2 \_1$ will be generated. In contrast, $\mathrm{E}_{\mathrm{p}}\left(r 1 \_p 1\right)=1^{\Upsilon}(\operatorname{cor}, x)$ will cause ERT for AP1 to contain one entry (Move $p 1$, request, $\left.1^{\prime}(\mathrm{car}, \mathrm{x})\right)$. 


\section{Appendix C1: Global Resource Table (GRT) Generation Algorithm}

Input: a list of ERT ( $A_{1}$.ERT, $A_{2}$.ERT, ...... $A_{n}$.ERT ), where $A_{i}$ is the ith agent in the MAS, $A_{i}$.ERT is the ERT provided by $\mathrm{A}_{\mathrm{i}}$

Output: a set of GRTs, where each GRT has a different owner

For $\mathrm{A}_{\mathrm{i}}$ ERT $(1<=\mathrm{i}<=\mathrm{n})$

For each entry $(t$, type, $P I)$

If (no such GRT $\mathrm{GR}_{\mathrm{i}}$ exists, where $\mathrm{GRT}_{\mathrm{i}}$.owner $=c$, where $c$ is the colored token or a colored token variable contained in PI)

Begin

Create a GRT for c;

Else

$$
\text { GRT.owner }=\mathrm{c}
$$

set GRT $=$ the existing GRT $\mathrm{G}_{\mathrm{i}}$; where $\mathrm{GRT}_{\mathrm{i}}$.owner $=c$, where $c$ is the colored token or a colored token variable contained in PI

End If

Add $\left(\mathrm{A}_{\mathrm{i}} \cdot\right.$ t, type, $\left.\mathrm{PI}\right)$ into $\mathrm{GRT}_{\mathrm{i}} \quad / /$ Prefix $\mathrm{A}_{\mathrm{i}}$ is added to transition $t$

End For

\section{Appendix C2: Comments and Examples for Global Resource Table (GRT) Generation}

After applying the resource table generation algorithm, we get a list of resource tables (GRTs), where each GRT is owned by a token color (a token color may contain variable, such as (car, $x$ ). Fig. 2 shows the list of GRTs for all the agents in the MAS Simple Com. Due to the fact that this example is simple, we only have one entry for each GRT. In Fig. 2, the generated GRTs contain variables. Also, note that the resource table generation algorithm only handles PIs with one token color. Processing ERT entries containing multiple different colors of tokens or token variables (for example, entry (Move_P1, release, 1 ' car +2 bus) contain two token colors, car and bus) would be more complicated, but follows the same principle as for handling inscriptions with only one token color or variable. Part of our future research is to handle inscriptions with multiple different color tokens. Currently, we only consider a PI that evaluates to one element.

\begin{tabular}{|c|c|}
\hline Owner & Entries \\
\hline$(\operatorname{car}, x)$ & (AP1.Move_P1, request, 1 (car, $x))$ \\
\hline (car, blue) & (AP2.Move_P1, release, 1`(car, blue)) \\
\hline (car, red) & (AP3.Move P1, release, 1 (car, red)) \\
\hline
\end{tabular}

Fig. 2. The Global Resource Tables for Simple Com 


\section{Appendix D1: Intermediate Coordinator Generation Algorithm}

Input: A list of GRTs $\left(\mathrm{A}_{\mathrm{i}} . \mathrm{t}\right.$, type, $\left.\mathrm{PI}\right)$

Output: A coordinator $C=\left(\Sigma_{c}, P_{c}, T_{c}, A_{c}, N_{c}, C_{c}, G_{c}, E_{c}, I_{c}\right)$

For each $\mathrm{GRT}_{\mathrm{i}}$

Create a color set RES for $\mathrm{GRT}_{\mathrm{i}}$. owner, and add the color set RES into $\Sigma_{\mathrm{c}}$

If (GRTi.owner contains no variable)

RES only contains one element GRTi.owner;

If (GRTi.owner contains variables)

RES contains all the possible assignment of GRTi.owner

Create place $\mathrm{p}_{\mathrm{i}}$ for the GRT $\mathrm{T}_{\mathrm{i}}$. Add $\mathrm{p}_{\mathrm{i}}$ into $\mathrm{P}_{\mathrm{c}}$

$\mathrm{C}_{\mathrm{c}}\left(\mathrm{p}_{\mathrm{i}}\right)=\mathrm{RES} ; / /$ define the color set for $G R T_{i}$.

For the jth element $\left(A_{i} . t\right.$, type, $\left.P I\right)$ in $G_{R T}$

Create transition $A_{i} \cdot t$, add $A_{i} \cdot t$ into $T_{c}$

Add $r_{i j}$ into $A_{c}$

If (type $==$ request)

$\operatorname{Add~} N_{c}\left(r_{i j}\right)=\left(p_{i}, A_{i} \cdot t\right)$

If (type $==$ release)

Add $N_{c}\left(r_{i j}\right)=\left(A_{i} \cdot T, p_{i}\right)$

Ec (rij ) = PI // Add inscription PI for $r$;

End For

End For

$\mathrm{G}_{\mathrm{c}}=\mathrm{G}_{\mathrm{T}}$

$\mathrm{I}_{\mathrm{c}}=\{\}$ (No initial resource, the coordinator designer can initialize it; this is not automated).

$\mathrm{C}=\left(\Sigma_{\mathrm{c}}, \mathrm{P}_{\mathrm{c}}, \mathrm{T}_{\mathrm{c}}, \mathrm{A}_{\mathrm{c}}, \mathrm{N}_{\mathrm{c}}, \mathrm{C}_{\mathrm{c}}, \mathrm{G}_{\mathrm{c}}, \mathrm{E}_{\mathrm{c}}, \mathrm{I}_{\mathrm{c}}\right)$, where:

$\Sigma_{\mathrm{c}}=\{\{($ car, blue $)\},\{($ car, red $)\}, \mathrm{CAR}\}$;

$\mathrm{P}_{\mathrm{c}}=\left\{p_{1}, p_{2,} p_{3}\right\}$

$\mathrm{T}_{\mathrm{c}}=\left\{\right.$ AP1.Move $p 1$, AP2.Move $p 1$, AP3.Move $\left.\_1\right\}$.

$A_{\mathrm{c}}=\left\{r_{11}, r_{21}, r_{31}\right\}$.

$N_{\mathrm{c}}=\left\{N_{\mathrm{c}}\left(r_{11}\right)=\left(\right.\right.$ AP2.Move $\left.p 1, p_{1}\right), N_{\mathrm{c}}\left(r_{21}\right)=\left(\right.$ AP3. Move $\left.p 1, p_{2}\right), N_{\mathrm{c}}\left(r_{31}\right)=\left(p_{3}\right.$, AP1. Move $\left.\left.p 1\right)\right\}$.

$\mathrm{C}_{\mathrm{c}}=\left(\mathrm{C}_{\mathrm{c}}\left(p_{1}\right)=\{(\right.$ car, blue $)\}, \mathrm{C}_{\mathrm{c}}\left(p_{2}\right)=\{($ car, red $\left.)\}, \mathrm{C}_{\mathrm{c}}\left(p_{3}\right)=\mathrm{CAR}\right)$.

$G_{\mathrm{c}}=G_{T}$

$E_{c}=\left\{E_{c}\left(r_{11}\right)=1\right.$ (car, blue $), E_{c}\left(r_{21}\right)=1$ (car, red $), E_{c}\left(r_{31}\right)=1$ (car, $\left.\left.x\right)\right\}$.

$\mathrm{I}_{\mathrm{c}=} \mathrm{I}_{\mathrm{AM}}=\left\{I\left(p_{1}\right)=0, I\left(p_{2}\right)=0, I\left(p_{3}\right)=0\right\}$.

Fig. 3. Textual Notation for an Intermediate Coordinator for Agents in Simple Com (Variables unresolved)

\section{Appendix D2: Comments and Examples for Intermediate Coordinator Generation}

Applying the intermediate coordinator generation algorithm to the GRTs in Fig. 2, we can generate the intermediate coordinator with the graphical diagram shown as Fig. 6.3 of Section 6 and the textual notation shown here in Fig. 3. The intermediate coordinator generation algorithm creates a color set for each GRT owner, a place for the same GRT owner, and assigns the newly created color set as the color set of the place. If a GRT owner has no variables, its color set contains only one element, the GRT owner itself. In contrast, the color set for a GRT owner with variables should contain all the possible assignments of this GRT owner. For example, a single element color 
set $\{$ (car, blue) $\}$ is created for GRT owner (car, blue) from Fig 2, and we also create place $\mathrm{p}_{1}$ for the same GRT owner, and assign $\{$ (car, blue) $\}$ as the color set of $\mathrm{p}_{1}$. The GRT owner (car, $x$ ) from Fig 2 contains variable $x$, which can be red or blue, so its color set is $\{(\mathrm{car}, \mathrm{red}) \cup(\mathrm{car}, \mathrm{blue})\}=\mathrm{CAR}$. The algorithm also creates a transition for each GRT entry, and connects the transition to the corresponding CPN place by arcs according to the type element in the GRT entry. For instance, we create the transition AP2.Move_P1 for the entry (AP2.Move_P1, release, 1Y(car, blue)) and connect it to place $\mathrm{p}_{1}$. Since the type element in entry (AP2.Move_P1, release, 1'(car, blue)) is release, we add arc $r_{11}$ from the transition AP2.Move_P1 to place $p_{1}$. It is also worth noting that $\mathrm{I}_{\mathrm{c}}$ generated by this intermediate coordinator generation algorithm is empty, but the coordinator can be post-processed to change $\mathrm{I}_{\mathrm{c}}$ to model the resources available initially.

\section{Appendix E1: Global Plan Generation Algorithm}

Input: (1) the set of path models $\mathrm{A}=\left\{\mathrm{A}_{\mathrm{i}}(1<=\mathrm{i}<=\mathrm{n}) \mid \mathrm{A}_{\mathrm{i}}=\left(\Sigma_{\mathrm{PM}}=\left(\Sigma_{\mathrm{r}}, \Sigma_{\mathrm{p}}\right), \mathrm{P}_{\mathrm{PM}}, \mathrm{T}_{\mathrm{PM}}, \mathrm{A}_{\mathrm{PM}}, \mathrm{N}_{\mathrm{PM}}, \mathrm{C}_{\mathrm{PM}}, \mathrm{G}_{\mathrm{PM}}\right.\right.$, $\left.\left.\mathrm{S}_{\mathrm{PM}}=\left(\mathrm{E}_{\mathrm{r}}, \mathrm{E}_{\mathrm{p}}\right), \mathrm{I}_{\mathrm{PM}}\right)\right\}$. Here $n$ equals the number of agents in the local plan.

(2) the coordinator $\mathrm{C}=\left(\Sigma_{c}, \mathrm{P}_{\mathrm{c}}, \mathrm{T}_{\mathrm{c}}, \mathrm{A}_{\mathrm{c}}, \mathrm{N}_{\mathrm{c}}, \mathrm{C}_{\mathrm{c}}, \mathrm{G}_{\mathrm{c}}, \mathrm{E}_{\mathrm{c}}, \mathrm{I}_{\mathrm{c}}\right)$.

Output: the global plan $G=\left(\Sigma_{g}, P_{g}, T_{g}, A_{g}, N_{g}, C_{g}, G_{g}, E_{g}, I_{g}\right)$

(all the places and transitions are already prefixed by agent id)

$\Sigma_{\mathrm{g}}=\cup \quad \mathrm{A}_{\mathrm{i} . .} \Sigma_{\mathrm{r}}(1<=\mathrm{i}<=\mathrm{n}) \cup \Sigma_{\mathrm{c}}$

$\mathrm{P}_{\mathrm{g}}=\cup \quad \mathrm{A}_{\mathrm{i} .} \mathrm{P}_{\mathrm{PM}}(1<=\mathrm{i}<=\mathrm{n}) \cup \mathrm{P}_{\mathrm{c}}$;

$T_{g}=\cup A_{i} \cdot T_{P M}(1<=i<=n) \cup T_{c} \cdot T_{b} ; / /$ Here $T_{c} \cdot T_{b}$ refers to the set of binding transitions in the coordinator, path transitions are excluded, because they have been merged to corresponding transitions in $A_{i} . T_{P M}$

$\mathrm{A}_{\mathrm{g}}=\cup \quad \mathrm{A}_{\mathrm{i} .} \mathrm{A}_{\mathrm{PM}}(1<=\mathrm{i}<=\mathrm{n}) \cup \mathrm{A}_{\mathrm{c}}$;

$\mathrm{N}_{\mathrm{g}}=\cup \quad \mathrm{A}_{\mathrm{i} .} \mathrm{N}_{\mathrm{PM}}(1<=\mathrm{i}<=\mathrm{n}) \cup \mathrm{N}_{\mathrm{c}}$;

$\mathrm{C}_{\mathrm{g}}=\cup \quad \mathrm{A}_{\mathrm{i} .} \mathrm{C}_{\mathrm{PM}}(1<=\mathrm{i}<=\mathrm{n}) \cup \mathrm{C}_{\mathrm{c}}$;

$\mathrm{G}_{\mathrm{g}}=\cup \quad \mathrm{A}_{\mathrm{i} .} \mathrm{G}_{\mathrm{PM}}(1<=\mathrm{i}<=\mathrm{n}) \cup \mathrm{G}_{\mathrm{c}}$;

$E_{g}=U A_{i} . E_{r}(1<=i<=n) \cup E_{c} ; / / A_{i} . E_{p}$ is not included in $E_{g}$, because the corresponding information have been collected by $\mathrm{E}_{\mathrm{c}}$

$I_{g}=\cup \quad A_{\text {i. }} I_{P M}(1<=i<=n) \cup \quad I_{c}$

\section{Appendix E2: Comments and Examples for Global Plan Generation}

Concatenation of local plans and a complete coordinator can be realized by merging corresponding transitions from the coordinator and local plans. When two transitions are merged, the arcs connected to a transition in a local plan would be connected to the corresponding transition in the coordinator. Also, the agent id is added as a prefix for those places and transitions in local plans to ensure the unique naming in the resulting global plan. Following the definition of global plan, we can concatenate path models of the three agents (Fig. 1) in Simple Com with the coordinator in Fig 5.4 of Section 5. The resulting global plan is shown in Fig. 4 with textual notation. 
$\Sigma_{\mathrm{g}}=\{($ car, blue $),($ car, red), per, CAR $\}$

$\mathbf{P}_{\mathrm{g}}=\left\{\mathrm{p}_{1}, \mathrm{p}_{2}, \mathrm{p}_{3}\right.$, AP1.Home, AP1.Office, AP2.Home, AP2.Office, AP3.Home, AP3.Office $\}$

$\mathbf{T}_{\mathbf{g}}=\{$ AP1.Move _p1, AP2.Move p1, AP3.Move_p1, t13, t31, t23, t32 $\} / / \mathrm{T}_{\mathrm{c}}$ is not included, because it has been merged to corresponding transitions in $\mathrm{A}_{\mathrm{i} .} \mathrm{T}_{\mathrm{PM}}$

$\mathbf{A}_{\mathbf{g}}=\left\{r_{11}, r_{21}, r_{31}, r 1, r 2, r 3, r 4, r 5, r 6, r 7, r 8, A P 1 . r 1 \_p 1, A P 1 . r 2 \_p 1, A P 2 . r 1 \_p 1, A P 2 . r 2 \_p 1, A P 3 . r 1 \_p 1, A P 3 . r 2 \_1\right\}$

$\mathbf{N}_{\mathbf{g}}=\{$

$N_{g}\left(r_{11}\right)=\left(\right.$ AP2.Move $\left.p 1, p_{1}\right)$,

$N_{g}\left(r_{21}\right)=\left(\right.$ AP3. Move $\left.p 1, p_{2}\right)$,

$N_{g}\left(r_{31}\right)=\left(p_{3}\right.$,APl.Move $\left.p 1\right)$,

$N_{g}($ APlrl_pl $)=($ AP1 Home, AP1.Move $)$,

$N_{g}($ AP1 $r 2$ p1 $)=($ AP1 Move, AP1 Office $)$,

$N_{g}\left(A P 2 r 1 \_1\right)=(A P 2$ Home, AP2 Move $)$,

$N_{g}(A P 2 . r 2 p 1)=(A P 2$ Move, AP2.Office) ,

$N_{g}\left(\right.$ AP1 $\left.r 1 \_p 1\right)=($ AP3 Home, AP3 Move $)$,

$N_{g}(A P 3 r 2 p 1)=($ AP3 Move, AP3.Office $)$,

$N_{\mathrm{c}}(r 1)=\left(p_{1}, t 13\right), N_{\mathrm{c}}(r 2)=\left(t 13, p_{3}\right), N_{\mathrm{c}}(r 3)=\left(p_{3}, t 31\right), N_{\mathrm{c}}(r 4)=\left(t 31, p_{1}\right), N_{\mathrm{c}}(r 5)=\left(p_{2}, t 23\right), N_{\mathrm{c}}(r 6)=\left(t 23, p_{3}\right)$,

$\left.N_{\mathrm{c}}(r 7)=\left(p_{3}, t 32\right), N_{\mathrm{c}}(r 8)=\left(t 32, p_{2}\right)\right\}$

$\mathbf{C}_{\mathrm{g}}=\{$

$\mathrm{C}_{\mathrm{g}}\left(p_{1}\right)=\{($ car, blue $)\}, \mathrm{C}_{\mathrm{g}}\left(p_{2}\right)=\{(\mathrm{car}, \mathrm{red})\}, \mathrm{C}_{\mathrm{c}}\left(p_{3}\right)=\mathrm{CAR}$,

$\mathrm{C}_{\mathrm{g}}($ AP1 Home $)=$ per, $\mathrm{C}_{\mathrm{g}}($ AP1 Office $)=$ per ,

$\mathrm{C}_{\mathrm{g}}($ AP2 Home $)=$ per, $\mathrm{C}_{\mathrm{g}}($ AP2 Office $)=$ per,

$\mathrm{C}_{\mathrm{g}}($ AP3 Home $)=$ per, $\mathrm{C}_{\mathrm{g}}($ AP 3 .Office $)=$ per $\}$

$\mathbf{G}_{\mathrm{g}}=\cup \quad \mathrm{A}_{\mathrm{i} .} \mathrm{G}_{\mathrm{PM}}(1<=\mathrm{i}<=\mathrm{n}) \cup \mathrm{G}_{\mathrm{c}}=\mathrm{G}_{\mathrm{T}}$.

$\mathbf{E}_{\mathrm{g}}=\{$

$E_{g}\left(r_{11}\right)=1^{\prime}($ car, blue $), E_{g}\left(r_{21}\right)=1^{\prime}($ car, red $), E_{g}\left(r_{31}\right)=1^{\prime}($ car, $x)$,

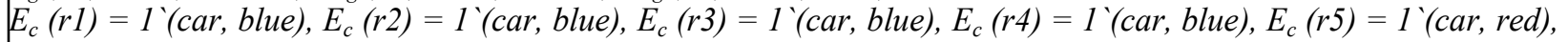

$E_{c}(r 6)=1$ (car, red), $E_{c}(r 7)=1$ (car, red $), E_{c}(r 8)=1$ (car, red $)$,

$E_{g}\left(A P 1 r 1 \_p 1\right)=1$ person,

$E_{g}(A P 1 r 2$ p 1$)=1$ person, $E_{g}(A P 2 r 1$ p1) $=1$ 'person,

$E_{g}\left(A P 2 r 2 \_1\right)=1$ person, $E_{g}\left(A P 3 r 1 \_p 1\right)=1$ person,

$E_{g}(A P 3 r 2$ pl) $=1$ person $\} / / \mathrm{A}_{\mathrm{i}} \mathrm{E}_{\mathrm{p}}$ is not included in $\mathrm{E}_{\mathrm{g}}$, because the corresponding information has been collected by $\mathrm{E}_{\mathrm{c}}$

$\mathbf{I}_{\mathbf{g}}=\{$

$\mathrm{I}_{\mathrm{g}}($ AP1.Home $)=1$ 'person, $\mathrm{I}_{\mathrm{g}}$ (AP1.Office $)=0$,

$\mathrm{I}_{\mathrm{g}}($ AP2.Home $)=1$ 'person, $\mathrm{I}_{\mathrm{g}}($ AP2. Office $)=0$,

$\mathrm{I}_{\mathrm{g}}($ AP3.Home $)=1$ 'person, $\mathrm{I}_{\mathrm{g}}($ AP3.Office $)=0$,

$\mathrm{I}_{\mathrm{g}}\left(p_{I}\right)=0, \mathrm{I}_{\mathrm{g}}\left(p_{2}\right)=0$ \}

Fig. 4. Textual Notation of the Global plan for Simple Com 